AperTO - Archivio Istituzionale Open Access dell'Università di Torino

\title{
Safeguarding and Accessing Drama As Intangible Cultural Heritage
}

\section{This is the author's manuscript}

Original Citation:

Availability:

This version is available http://hdl.handle.net/2318/1556751

since 2016-11-29T15:23:27Z

Published version:

DOI:10.1145/2812814

Terms of use:

Open Access

Anyone can freely access the full text of works made available as "Open Access". Works made available under a Creative Commons license can be used according to the terms and conditions of said license. Use of all other works requires consent of the right holder (author or publisher) if not exempted from copyright protection by the applicable law. 
This is the author's final version of the contribution published as:

Lombardo, Vincenzo; Pizzo, Antonio; Damiano, Rossana. Safeguarding and Accessing Drama As Intangible Cultural Heritage. ACM JOURNAL ON COMPUTING AND CULTURAL HERITAGE. 9 (1) pp: 1-26. DOI: $10.1145 / 2812814$

The publisher's version is available at:

http://dl.acm.org/citation.cfm?doid=2846105.2812814

When citing, please refer to the published version.

Link to this full text:

http://hdl.handle.net/2318/1556751 


\title{
Safeguarding and Accessing Drama as Intangible Cultural Heritage
}

\author{
VINCENZO LOMBARDO, ANTONIO PIZZO, and ROSSANA DAMIANO, University of Torino
}

\begin{abstract}
Storytelling, especially in the form called drama, is pervasive across cultures and ages. Though much attention has been devoted to the preservation of the physical supports of drama (e.g., films and tapes), there is a widespread acknowledgement that such tangible heritage is the expression of an intangible notion of drama. This paper introduces the drama as a form of intangible cultural heritage and presents a solution for its preservation in terms of a formal encoding through a computational ontology.

After the review of a formal representation of drama, called Drammar, developed in previous works, we show how an abstraction of drama can be encoded into a digital item. We also show how the method proposed is compliant with the major initiatives for the documentation of cultural heritage, namely CIDOC-CRM and FRBR. Finally, we test the applicability of our solution by showing how the major tenets of two well-known theories of drama can be encoded in Drammar and presenting the results of a focus group of drama scholars and practitioners who have accessed the encoding through a visualization system. The encoding and visualization system have turned out to be a promising support for teaching and investigating drama.
\end{abstract}

CCS Concepts: $\bullet$ Applied computing $\rightarrow$ Performing arts; Digital libraries and archives; $\bullet$ Computing methodologies $\rightarrow$ Ontology engineering;

Additional Key Words and Phrases: drama heritage, intangible cultural heritage, digital heritage, CIDOC-CRM, computational ontology

ACM Reference Format:

Vincenzo Lombardo, Antonio Pizzo, and Rossana Damiano. 2015. Drama as Intangible Cultural Heritage. ACM J. Comput. Cult. Herit. 2, 3, Article 1 (May 2010), 27 pages.

DOI : 0000001.0000001

\section{INTRODUCTION}

This paper addresses the cultural construct known as drama. A drama item is a story conveyed through characters who perform live actions: Shakespeare's Hamlet as well as Tom Stoppard's Rosencrantz and Guildenstern Are Dead, the HBO's Sopranos, even most reality shows, such as CBS's Survivors, and, finally, Rockstar Games' L.A. Noir or Ubisoft's Assassin's Creed series. Drama has been pivotal for storytelling across all cultures and ages [Mamet 1998]: it has grown through different media [Esslin 1988] and has been most pervasive, from theatre and cinema to TV and videogames. Along these media, a single drama can assume several forms, fulfilling a number of its core conditions. For example, the abstraction of the oral tale Cinderella has, e.g., Perrault's and Disney's versions.

Abstracting from the media objects that exhibit a drama, we face a form of intangible cultural heritage (ICH), as we show in Section 2. Our goal is to build a digital item of such a story abstraction, in

\footnotetext{
Author's address: Vincenzo Lombardo, CIRMA and Dipartimento di Informatica, Università degli Studi di Torino, C.so Svizzera, 185 - 10149 Torino (Italy); email: vincenzo.lombardo@unito.it; Antonio Pizzo, CIRMA and Dipartimento di Studi Umanistici, Università degli Studi di Torino, Palazzo Nuovo, Via Sant'Ottavio 20 - 10124 Torino (Italy); email: antonio.pizzo@unito.it. Rossana Damiano, CIRMA and Dipartimento di Informatica, Università degli Studi di Torino, C.so Svizzera, 185 - 10149 Torino (Italy); email: rossana.damiano@unito.it.

Permission to make digital or hard copies of all or part of this work for personal or classroom use is granted without fee provided that copies are not made or distributed for profit or commercial advantage and that copies bear this notice and the full citation on the first page. Copyrights for components of this work owned by others than ACM must be honored. Abstracting with credit is permitted. To copy otherwise, or republish, to post on servers or to redistribute to lists, requires prior specific permission and/or a fee. Request permissions from permissions@ acm.org.

(C) 2010 ACM. 1556-4673/2010/05-ART1 $\$ 15.00$

DOI : 0000001.0000001
} 
order to enhance the possibilities of preservation. ${ }^{1}$ One of the key challenges is the sharing and consensus on terms: the working hypothesis in this paper is that computational ontologies and semantic web technologies can fulfill such requirement. The digital item must be expressed in machine-readable formats, in order to limit, as far as possible, terminological ambiguities and vagueness and support accessibility and preservation.

In previous research, we have addressed the problem of metadata annotation for dramatic media through the introduction of a drama ontology, called Drammar [Lombardo et al. 2014] [Lombardo and Pizzo 2014]. Drammar encodes the major concepts and relations of the drama domain, the so-called dramatic qualities, which have been shared by a majority of scholars in the drama literature, and provides the terminological knowledge for the instantiating the annotation metadata for the dramatic media objects. In this paper, we employ Drammar as a tool for building a digital item that encodes the intangible heritage of some drama. Section 3 surveys the elements of drama domain and the Drammar encoding. In addition to review the major Drammar tenets, we also discuss how Drammar can be positioned within the framework of ontologies currently employed for the documentation of cultural heritage, namely CIDOC CRM and FRBR (Section 4). As we will see, the digital item that preserves drama as a form of intangible cultural heritage is an expression of an abstract dramatic work in the formal language of the computational ontologies.

The practical applicability of our method to the preservation of drama is then demonstrated through the definition of a workflow for the encoding of a drama (Section 5) and the exemplification of an encoding for a relevant scene from Hamlet.

Finally, we test the usability of the proposed of the ontology encoding through two approaches (Section 6). The first approach aims at demonstrating that Drammar is able to encode two exemplary statements about drama: (a) the drama as a specific narrative structure, as described by Freytag's triangle; (b) the drama as a type of interaction over a set of characters, as in Polti's 36 dramatic situations. The second approach consists in running a focus group with experts and practitioners aimed at assessing the readability of the ontology-based visualization of drama and of its usefulness in specific tasks.

In Section 7, we survey a number of approaches to the annotation of drama and the use of computational ontologies in multimedia that, in a broad sense, have inspired some aspects of our work. Conclusion ends the paper.

\section{THE CASE FOR DRAMA AS INTANGIBLE CULTURAL HERITAGE}

The notion of Intangible Cultural Heritage (ICH) is still debated. As clearly stated in the Preamble of the 2003 Convention [UNE 2003], there exists an interdependence between the intangible cultural heritage and the tangible cultural heritage. Thus, it is quite impossible to define something intangible without referring to something tangible. We face a complex scenario of interlinked notions, where there are items to be safeguarded as Natural or Cultural Heritage, although these items are also the manifestations of a Intangible Cultural Heritage that has to be represented and safeguarded. If we take drama as $\mathrm{ICH}$, these manifestations are the existing dramatic media items, and we need to devise a digital representation to bring upfront the social and symbolic values [Smith and Akagawa 2008].

In its wider meaning, drama heritage can incorporate, e.g., the performances of the Greek tragedies as well as those of Ibsen's theatrical works, together with the most recent Hollywood movies; also, drama is not intended for some specific form of manifestation (a manuscript, a book, a film, etc.), and we can generally speak of "dramatic media" to signify that drama spans over multiple media [Esslin

\footnotetext{
$\overline{{ }^{1} \mathrm{http} / / / w w w . d p c o n l i n e . o r g / a d v i c e / p r e s e r v a t i o n h a n d b o o k / i n t r o d u c t i o n / d e f i n i t i o n s-a n d-c o n c e p t s ~}$
} 
1988]. All these items, which share the cultural construct that we recognize as drama, constitute a large body of evidence for drama studies and have been increasingly relevant since the availability of digital media. In the following, we enumerate the characteristics that make drama Intangible Cultural Heritage (cf. the enumeration provided by R. Smeets in [Smeets 2004, pp. 146-148]):

(1) Frequent absence of link with specific location: drama is something that can be performed but does not reside within a specific location; as it happens with music, drama can be performed in different locations and by different artists.

(2) Human borne, hence mobile and ephemeral: the knowledge to recreate drama and the regulatory system from where the necessary skills are activated are in the human mind, and the main means of expression of the drama are the actions of some embodied agent; although it can be transmitted in the traditional form of a text, this eminently bears the specific kind of instruction for some action to be performed; the elements of drama do not have a historically correct form or a historically correct function or meaning and may be reinterpreted as it normally happens in our Western culture (think of how many versions of Hamlet exist);

(3) Limited in duration and evolving: virtually, we cannot have two manifestations of a specific drama that are totally identical; but, also, the form and function of what we call drama may change; think of the difference between the Greek Tragedy Oedipus and the modernist play Six Characters in Search of an Author (in the two manifestations, also the drama (i.e. ICH) element is largely different).

(4) Transmitted from generation to generation: since drama is constantly evolving, it has to be transmitted and survives as skills and techniques, learnt by means of mimetic techniques by future generations; young authors study drama through the experience of the manifestations of the intangible heritage that we know as drama, being such experiences reading a text, attending a performance, watching a movie, listening to a radio drama, etc.

(5) Often spread over large areas or dispersed: many elements of the drama can be found spread and dispersed geographically and culturally, such as e.g., the original movie The Seven Samurai and the Hollywood movie The Magnificent Seven.

(6) Not safeguarded as living heritage by means of documentation: drama is documented in many different ways (text, score, video, audio, etc.); that is, one discrete manifestation of a drama is documented and, if the drama lives and continues to develop, such a documentation will have historical value, help research, memory, and transmission; researchers can consider such digital media items either as the direct object of study (in the case of, e.g., a movie) or as the video documentation of some theatre performance. However, such a documentation does not contribute to the safeguarding of the drama as an ICH item and scholars foresee a collaborative environment for the creation/sharing/dissemination of the metadata that express knowledge on the essential elements of drama and theatre [Carson 1997].

\section{THE DOMAIN OF DRAMA AND ITS ONTOLOGICAL REPRESENTATION}

In this section, we summarize the major tenets of the Drammar ontology, from the identification of a set of necessary elements that constitute the notion of drama to the encoding of such elements into a computational ontology (for more detail, see [Lombardo et al. 2014]).

Drama is a specific way to represent a story. Elam names the notion of story as fabula, an abstraction of the sujzet/plot [Elam 1980, p. 120]; Pfister concludes that "a number of different dramatic texts can be based on one and the same story and also that the same story may even be presented as texts in different media" [Pfister 1991, p. 197]. These varieties of texts and shapes are defined by Ryan as 
the several avatars of a story [Ryan 2006, p. xviii]; our model aims at addressing the specific kind of story encompassed by the notion of drama; and we adopt a technical point of view, such as that found in playwriting techniques [Egri 1946]. The Greek word drama is related to the notions of $d o$, act. Nowadays drama can be seen as a sequence of structured actions described in a text or in a score, regardless of the media and channels of the presentation (scores or, directly, software programs are the typical texts of interactive drama). Szondi has defined the drama as the action at the present time acted directly by characters [Szondi 1983, pp. 194-196]. Nevertheless, drama is "dramatic" because of its specific tools of mimesis, hence its specific language of actions [Aristotle 2013, 1453b 1-10] [Aristotle 2008, p. xxxviii]. Scholars have clearly stated that drama is made of characters' behaviors rather than its presentation in front of an audience [Brooks and Heilmann 1946], and that a dramatic action is not "doing something" but is achieving "what a character wants" [Spencer 2002, p. 38]. The action has to spread out of the character's inner motivation or has to be the character's reaction to some external pressure. The actions (and/or the reactions) must be consistent with the character's personality and intentions, which must be revealed through some clues; most importantly, the actions must produce high-level conflicts and the consequent emotions.

So, to explore the common dramatic qualities that reconcile Romeo and Juliet and Assassin Creeds, we focus on: 1) how the plot develops and how it is structurally organized, 2) how the characters deliberate and how they are engaged in the actions, 3) what conflicts take places, 4) what emotions spur from these conflicts. These qualities can be further specified as a set of dramatic elements, as found in drama analysts such as Lavandier [Lavandier 1994], Ryngaert [Ryngaert 2008], Hatcher [Hatcher 1996], and Spencer [Spencer 2002] who provide a syntax of the dramatic elements. These elements are embedded into the tangible heritage item (the item that concretely instantiates the drama) and constitute, in our approach, the intangible component of the cultural object. They are well established notions rooted both in the authors' craftsmanship and in the drama technique literature. We provide a method to interconnect them into a single conceptual framework, a computational ontology, the key to represent and safeguard such dramatic elements.

In order to build a formal encoding of the dramatic elements, Drammar resorts to a set of theories and models well established in Artificial Intelligence and Computer Science. As it will be clear in what follows, the ratio of this design strategy is twofold: on the one side, it relies on widespread, sound models, with formal properties that have been investigated in depth; on the other side, it augments the interoperability of the representation with other encodings, especially if obtained with the contribution of Artificial Intelligence, as in the case of systems for interactive storytelling, procedural animation, drama annotation.

The design of Drammar ontology relies on three representation layers (see Figure 1 for a synoptic overview). The first, the closest to the tangible drama item, is the observable timeline (top of Figure 1), appraised through a literary text or an audiovisual medium, a succession of the incidents (or actions) that happen in the drama. Incidents are assembled into discrete structures, called units. This level is formalized through the Situation Calculus paradigm [McCarthy 1986]: with units that function as operators advancing the story world from one state to another. The actions result from the deliberation process of the characters, named agents here (see below).

The deliberation process is represented by the motivational layer, which centers upon the notion of the character's intention in achieving (or trying to achieve) a goal. The intention, or the commitment of the character, is represented by a plan, which consists of the actions that are to be carried out in order to achieve some goal; plans are organized hierarchically, with high-level behaviors formulated as lower-level plans (or subplans). Goals originate from the values of the characters that are put at stake and need to be restored, given the beliefs (i.e. the knowledge) of the agents. This level is formalized through the rational agent paradigm, or BDI (Belief, Desire, Intention) paradigm [Bratman 1987] 


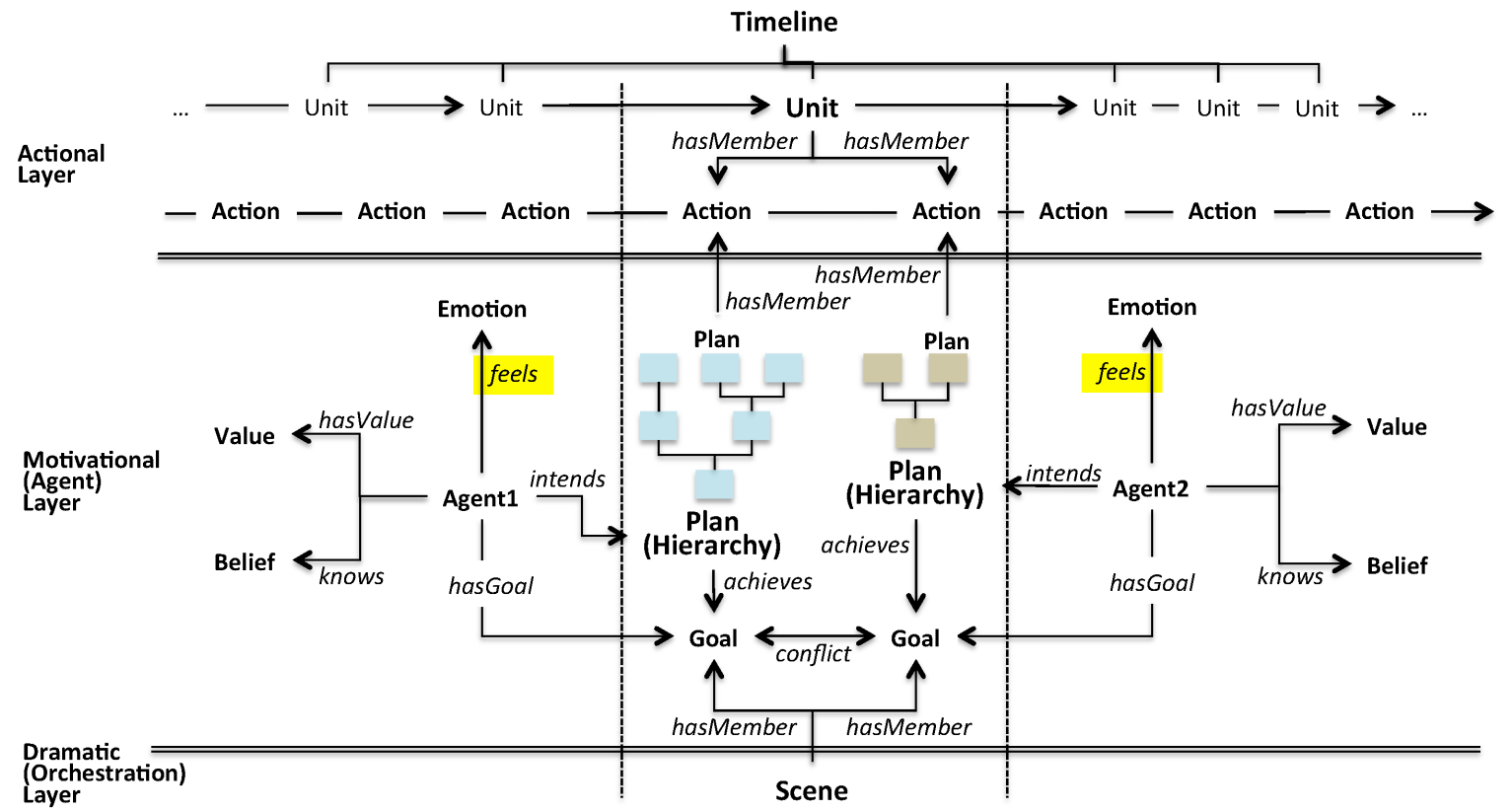

Fig. 1. Layers of ontology Drammar

(which has already seen some applications in the computational storytelling community [Norling and Sonenberg 2004] [Peinado et al. 2008]). This is why characters are encoded as agents in Drammar (middle of Figure 1). The plan is the major structure of the Motivational Layer, where all the other entities participate [Fikes and Nilsson 1971]; plan hierarchies are trees of plans, with mother plans that recursively dominate children subplans [Sardina et al. 2006], until basic plans with actions that are actually performed by the agents in the drama.

The dramatic layer, which is directly inspired by the literature on drama theory, accounts for the orchestration of the conflicts between the agents through the interplay of the actions to be assembled in units. The major structure here is the scene (bottom of Figure 1), which assembles several plans of different agents trying to achieve goals in conflict. The success/failure in achieving goals as well as in supporting own values is responsible for agent's appraisal of emotions. The vertical dashed bars, spanning the three layers in Figure 1, show how the scene determines the contents of a unit, by assembling the actions that are parts of plans aimed at achieving goals in conflict.

All the elements of the drama domain are encoded as classes and relations of a formal ontology consisting of logic-based descriptions. In formal ontologies, classes represent entity types (e.g., the notion of artwork may be represented by the Artwork class in a hypothetical ontology of art) and are arranged in a hierarchy from the most general to the more specific (e.g., Painting or Sculpture). Classes, at any level of the hierarchy, contain sets of exemplars, or individuals (e.g., Picasso's painting entitled Guernica may be represented by an individual belonging to the Artwork class). Relations, or properties, are defined over pairs of classes, and are instantiated on the individuals that populate the ontology: for example, the individual named Guernica may be connected to the individual named Picasso (instance of the Artist class) by the "painted by" relation. A class may also have properties that 
attach data to the individuals belonging to it, such as the creation date or the country of origin for an artwork.

The top level classes of the Drammar hierarchy are:

-DramaEndurant, which includes agents and objects that participate into actions (what distinguishes agents is intentionality) and "endure" their existence across the actions they participate into;

-DramaPerdurant, which includes processes (a superclass of actions) and states, distinguished through the dynamic/static dichotomy;

-DramaStructure, which includes those structures that organize the actions in ways that build a drama; in particular, structures include 1) the motivational structure (here called plan) that relates some goal of the an agent with the actions the agent committed to in order to achieve such goal, 2 ) the interplay structure (here called scene) that composes goals and plans of several agents to produce the conflicts, 3 ) the resulting segments that compose the whole drama (here called units), containing the drama incidents.

The abstract ontology, expressed as a set of logical specifications of classes and properties, needs to be expressed into a formal language to become a digital, textual artifact that can be fed to a software program (for manipulation, querying, comparison, etc.). The ontology languages designed as part of the Semantic Web project allow conceptual models to be described in an unambiguous way, open to understanding and manipulation by both human users and software programs. The concepts and relations introduced above are encoded in the ontology Drammar, written in the Semantic Web language known as OWL (Ontology Web Language). In particular, Drammar is written in a specific sub-language, OWL2 RL (Rule Language), which provides the adequate tradeoff between expressivity and complexity with respect to the requirements of the drama domain (see [Guarino et al. 2009] for an introduction to computational ontologies). On the one hand, the OWL component represents the concepts in a taxonomic structure and the relations by connecting the concepts; on the other, the rule-based RL component provides the conditional (IF-THEN) reasoning mechanisms that implement the inter-layer projection/appraisal operations. The classes and properties of the Drammar ontology provided a formalized model of the dramatic elements, directly expressible in OWL, namely Action (3.1), Agent (3.2), Structure, with reference to Unit in particular (3.3), Conflict, with the resulting Emotion (see Figures 4 and 9). Also, we have developed classes that are intended as an interface between the drama domain concepts and the linguistic and common sense types of knowledge that express the content of the drama, under the paradigm of linked data [Heath and Bizer 2011]. Having reviewed the ontological representation of the dramatic elements, we introduce a workflow for the semantic annotation of a tangible dramatic item.

In order to clarify the description, all along the paper we make reference to a running example taken from Shakespeare's Hamlet: the so called "nunnery" scene. In this scene, situated in the Third Act, Ophelia is sent to Hamlet by Polonius (her father) and Claudius (Hamlet's uncle, the king) to confirm the assumption that Hamlet's madness is caused by his rejected love. According to the two conspirators, Ophelia should induce him to talk about his inner feelings. At the same time, Hamlet tries to convince Ophelia that the court is corrupted and that she should go to a nunnery. In the middle of the scene, Hamlet puts Ophelia to a test to verify her honesty: guessing (correctly) that the two conspirators are hidden behind the curtain, he asks the girl to reveal where her father Polonius is. She decides to lie, by replying that he is at home. Hamlet realizes from the answer that also Ophelia is corrupted and consequently becomes very angry, realizing that there is no hope to redeem the court. The representation of the scene provided to exemplify the use of Drammar describes the excerpt in which Hamlet is testing Ophelia's honesty by asking rhetorically a question he knows the answer of, 
namely the current location of her father Polonius (the same room where they are, behind a curtain), and Ophelia lies by giving a false location, namely Polonius' home.

Here below, we describe the most significant classes and relations that form a drama: actions (a subclass of DramaPerdurant), agents (a subclass of DramaEndurant), and structures (class DramaStructures, with subclasses for plans, units, timeline, scenes). In the next subsections, we review the major definitions and refer to the published ontology for the formal format of classes ${ }^{2}$. Then, we address the practical task of building a digital item for some drama and test the usability of the representation.

\subsection{Action}

From Aristotle to Szondi, it is clear that drama is a specific manner of organizing actions that exhibit some qualities. Drammar splits the notion of dramatic action into intentional activities and states of the world that holds before or/and after the activities, namely processes and states, respectively. The distinction between processes and states on the one side, and agents and objects, on the other side, (see following Section 3.2), is inspired by the Endurant and Perdurant distinction in DOLCE ontology, while the representation of how characters (and objects) take part in processes and states with different roles, relies on the ontology design patterns, also defined on the top of DOLCE ontology [Gangemi and Presutti 2009], which abstract the participation of individuals to processes onto high level, re-usable and interoperable schemata. An individual of the class Action ${ }^{3}$ (see Figure 2) is an intentional process, that is, a process to which an individual of the class Agent is committed; such intentionality is expressed through the relation of containment of the action within an individual of the class Plan, committed by the agent (property intends). In order to be part of the drama, the action is also contained within an individual of the class Unit, that is there exists a relation of containment between an action and a unit. Figure 2 summarizes the representation, also instantiating an individual action. Here is a partial snippet of the OWL representation in RDF/XML triple serialization (cf. the names of the elements with the ones in Figure 2). The representation format shows the individual or instance (in the first line), with the relations that depart from that individual (subsequent lines): for example, the type of the individual A_ask_01 is the class Action, while the individual A_ask_01 is member of the unit Unit18_WQ.

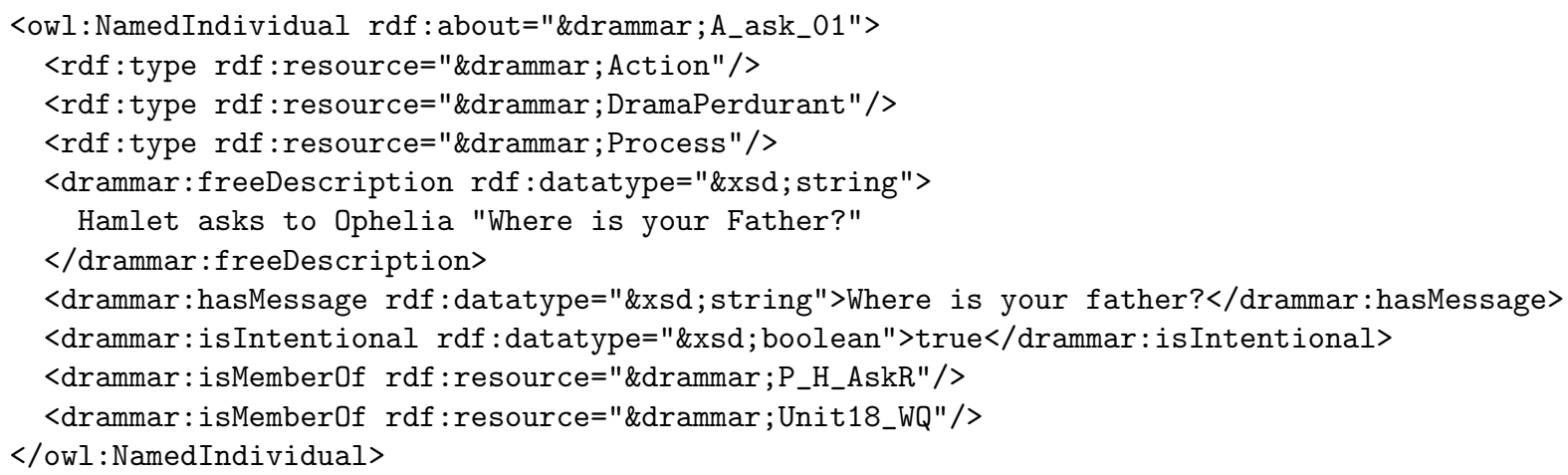

One individual action is described by a ProcessSchema ${ }^{4}$. This schema, pivoting the Time Indexed Situation design pattern [Gangemi and Presutti 2009], is mapped onto the linguistic description, in

\footnotetext{
${ }^{2}$ http://www.di.unito.it/ vincenzo/FTP_SWJ/drammar_info.html

${ }^{3}$ http://www.di.unito.it/ vincenzo/FTP_SWJ/drammar_info.html\#d4e1760

${ }^{4} \mathrm{http} / / /$ www.di.unito.it/ vincenzo/FTP_SWJ/drammar_info.html\#d4e3309
} 


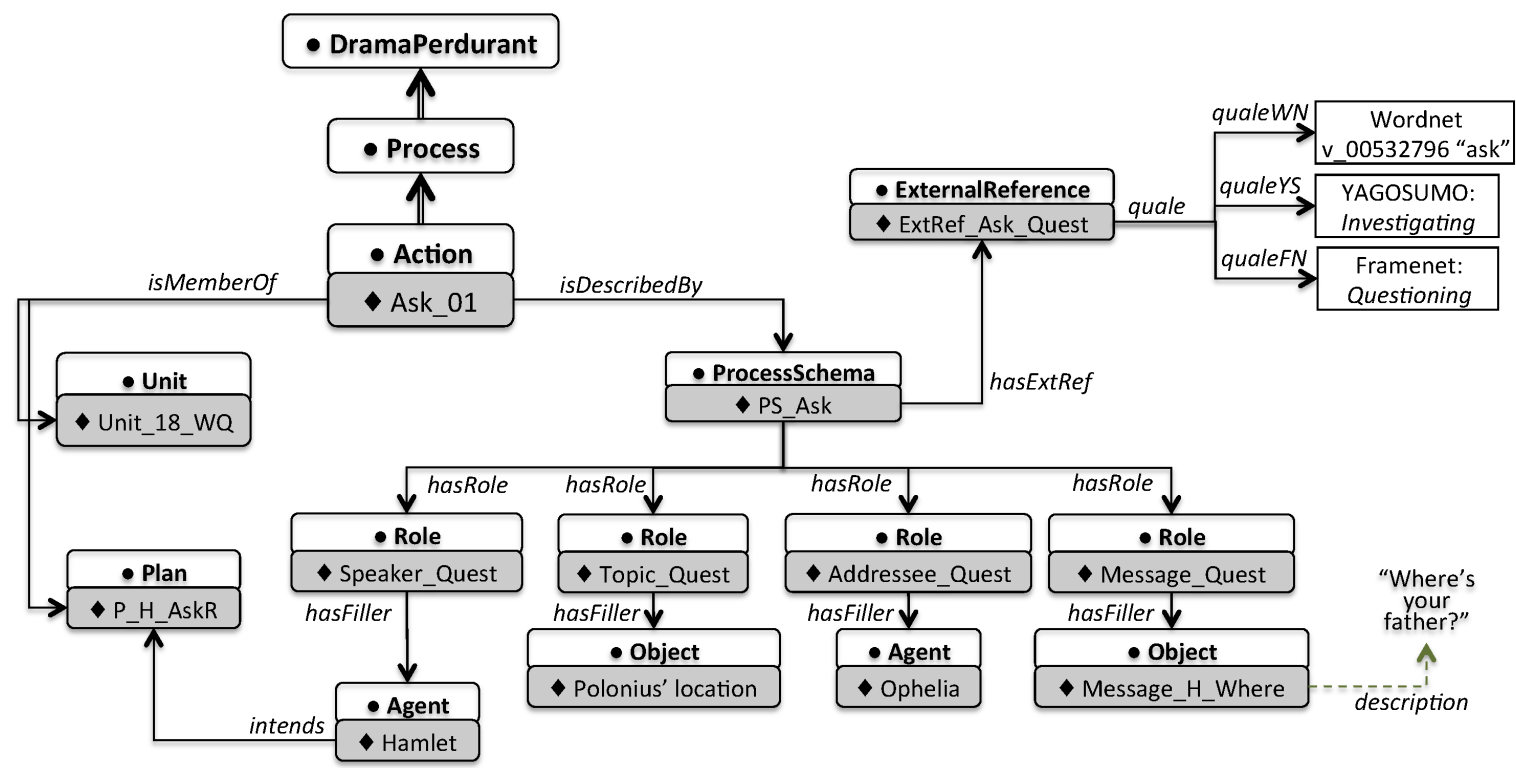

Fig. 2. The concept Action in the Drammar ontology, together with some significant properties. Subclass relations are represented with a double-line arrow. White boxes represent classes; grey boxes, associated with white boxes are instances of the classes.

terms of a role structure that is provided by the linguistic frames of FrameNet [Baker et al. 1998], indexed through the terms of the multilingual interface provided by MultiWordNet [Pianta et al. 2002] the cross-language version of the WordNet lexical data base [Miller 1995] (see the meaning annotation process in [Cataldi et al. 2013]). Drammar refers to two external large-scale semantic resources for the description of the common sense knowledge, namely the two ontologies Suggested Upper Merged Ontology (SUMO, [Pease et al. 2002]) and Yet Another Great Ontology (YAGO [Suchanek et al. 2007]), merged into YAGO-SUMO [De Melo et al. 2008], which provide very detailed information about millions of situations, including entities (agents and objects) and processes/actions. In Figure 2, we can see the linguistic term in Wordnet, the frame schema in Framenet, the semantic concept in YAGO-SUMO, for the action of Hamlet asking Ophelia about Polonius' location through the question "Where is your father?". In particular, the roles engaged by the frame are connected through the property instantiations hasRole to the process schema, and in turn the roles are connected to the agents and objects that are their fillers (property hasfiller). Also, note that Hamlet is both the speaker of the asking action and the agent that intends the plan that contains the action. In the system implemented for the annotation [Lombardo and Pizzo 2014], the terms in YAGO-SUMO are accessed through the lexical interface to increase the interoperability of the annotation.

\subsection{Agent}

The agent is a willing dynamic entity that constantly appraises the state of its world and acts after a rational deliberation that also implies an emotional charge. The agents in Drammar (see Figure $3)^{5}$ are represented as instances of the Agent class, subclass of DramaEndurant, have mental states

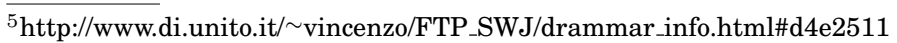




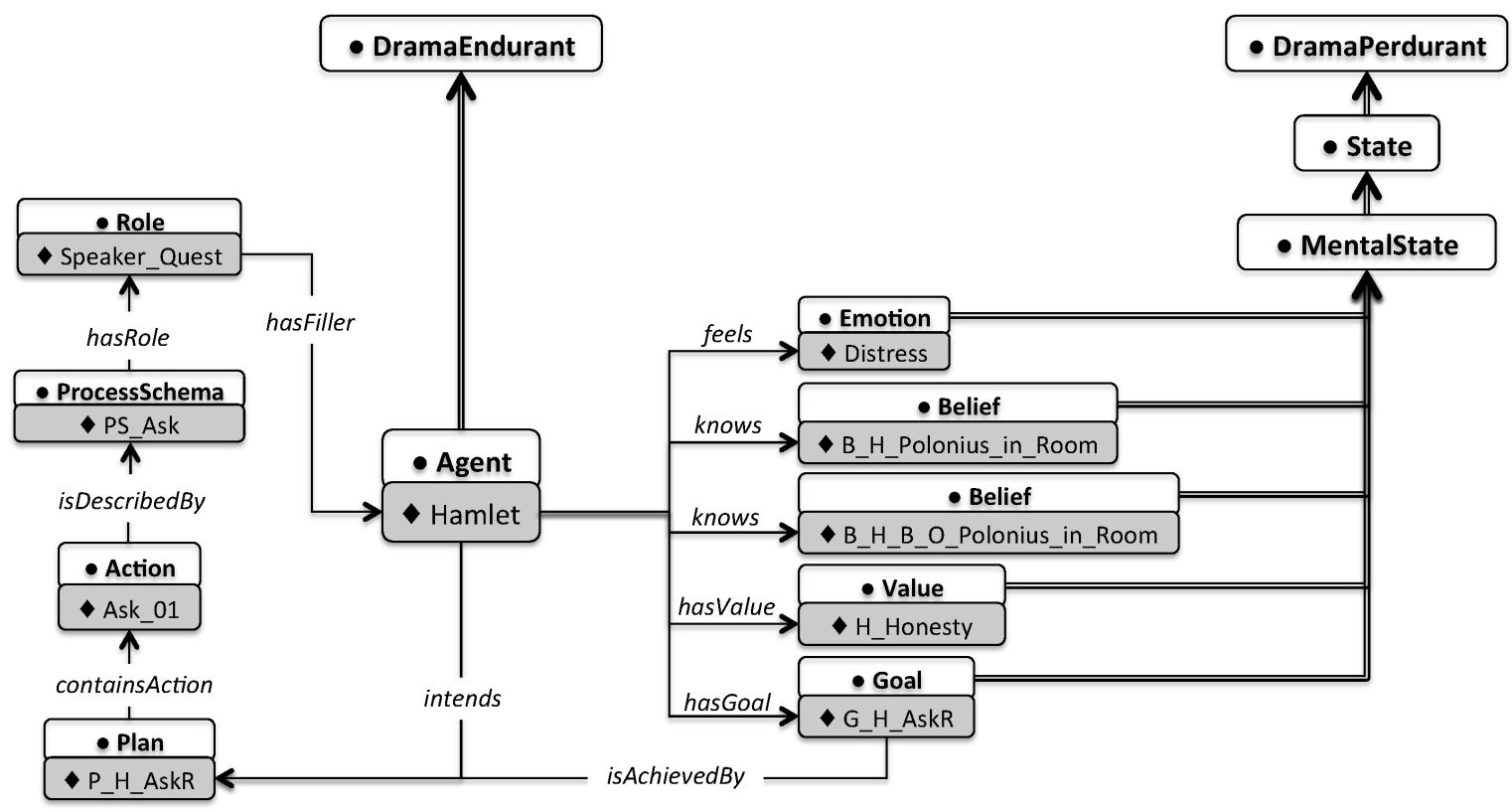

Fig. 3. The concept Agent in the Drammar ontology, together with some significant properties. Subclass relations are represented with a double-line arrow. White boxes are classes; grey boxes, associated with white boxes are instances of the classes.

(MentalState class), commit (property intends) to their plans of behavior (Plan class) to achieve their goals, and participate into actions as filler (property hasFiller) of a role (class Role) that is envisaged (property hasRole) by the relative process schema (class ProcessSchema).

The class MentalState ${ }^{6}$ features the following subclasses: Belief (the agent's subjective knowledge about the world, property knows); Emotion (what the agent feels, property feels); Goal (the objectives that motivate the actions of the agents, property hasGoal); Value (the moral values acknowledged by an agent; values can be put at stake or balanced (instances of the class ValueEngaged) by the unfolding of the story incidents). Plans are intended by agents (property intends) to achieve some goal (property achieves). So, in order to represent the interplay of actions and intentions, we resorted to a model of agency that ties intentions to actions into a unifying perspective.

In Figure 3, the agent Hamlet fills the speaker role of the asking action contained in one of his plans (P_H_AskR), the one of asking a rhetorical question. In fact, Hamlet knows that Polonius is in the room (a Belief B_H_Polonius_in_Room) and also knows that Ophelia knows that Polonius is in the room (another Belief B_H_B_O_Polonius_in_Room); Hamlet honesty (Value H_Honesty) is put at stake by Ophelia behavior (engaged value, not in the Figure). Since Hamlet knows the presumed truth (Polonius really is in the room), he formulates the Goal of posing a rhetorical question to Ophelia (Goal G_H_AskR), potentially achieved through a plan (P_H_AskR). As we will see below, the interplay of such plan with Ophelia's plan of lying about Polonius' location (she replies "At home, my lord.") causes distress to Hamlet (Emotion Distress connected through the feels property). Here is a partial snippet of the OWL representation for the agent Hamlet (it includes all the beliefs, goals, values and plans of Hamlet in the scene, represented, respectively by the properties knows, hasGoal, hasValue, and intends):

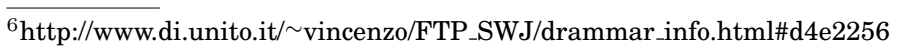




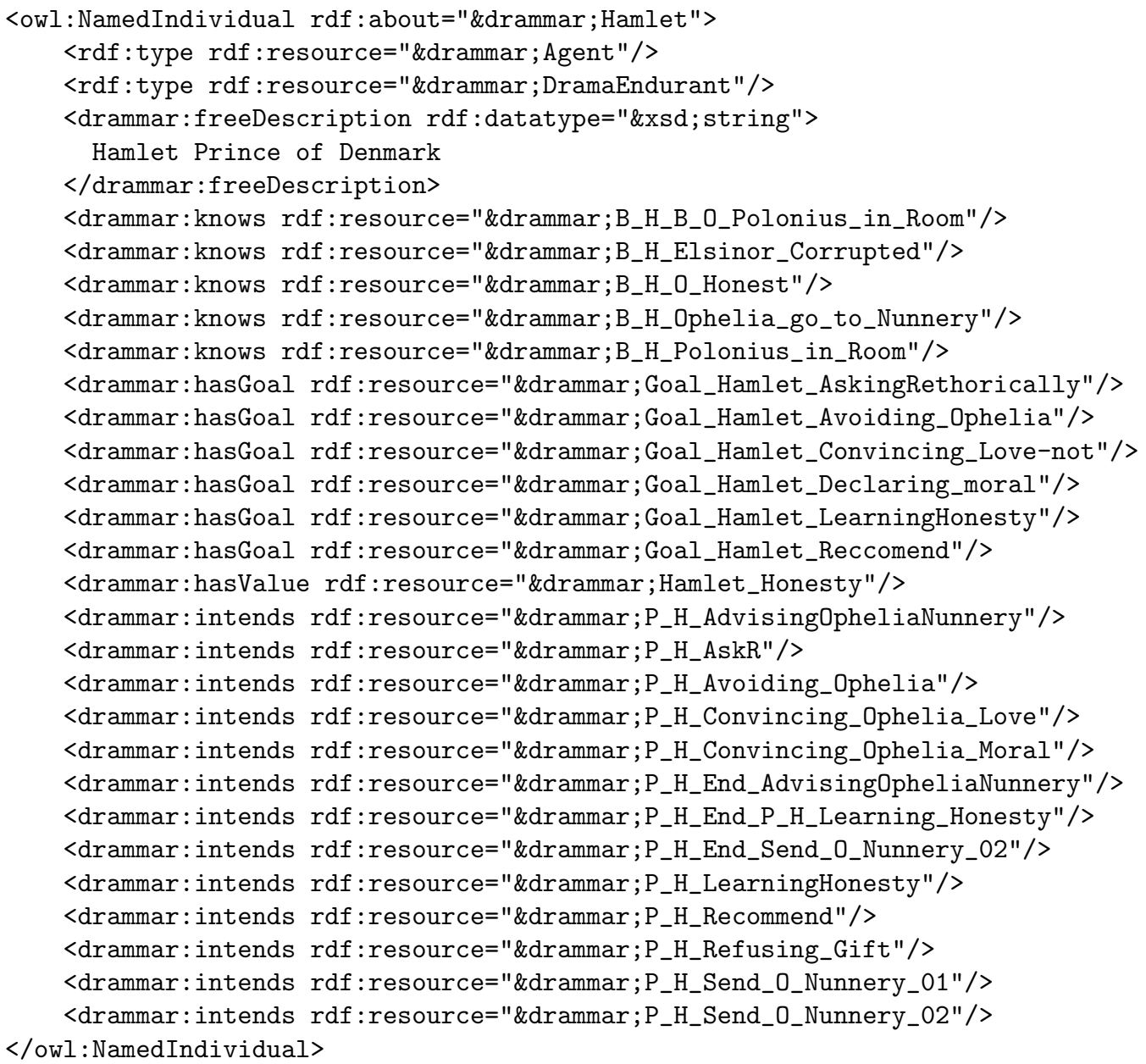

\subsection{The drama structures}

The essence and wholeness of drama springs from its parts. The elements listed above, i.e. actions and agents, must be coordinated within some container that allows them to interact with one another. In Drammar, such containers are all instances of the class DramaStructure, split into several subclasses. The units are the containers of the character's actions that result from the characters' deliberations. Units are assembly of actions that are put together for dramatic reasons. In particular, each agent carries on her/his plans of actions for achieving her/his goals; these goals are in conflict with one another; the interplay of the plans of actions that try to achieve goals in conflict are structured as scenes, which determine the units; a sequence of units is the timeline of the whole drama.

All the incidents (class Action) in the timeline (class Timeline) are contained in individuals of the class Unit (see Figure 4). So, a timeline is the chain of all the units (connected through the property precedes). Each unit has two unit states (i.e. two individuals of the UnitState class) associated: the unit state that precedes the unit (connected through the property hasUnitPrecondition) contains the states that hold in the story world before the incidents in the unit occur; the unit state that follows 


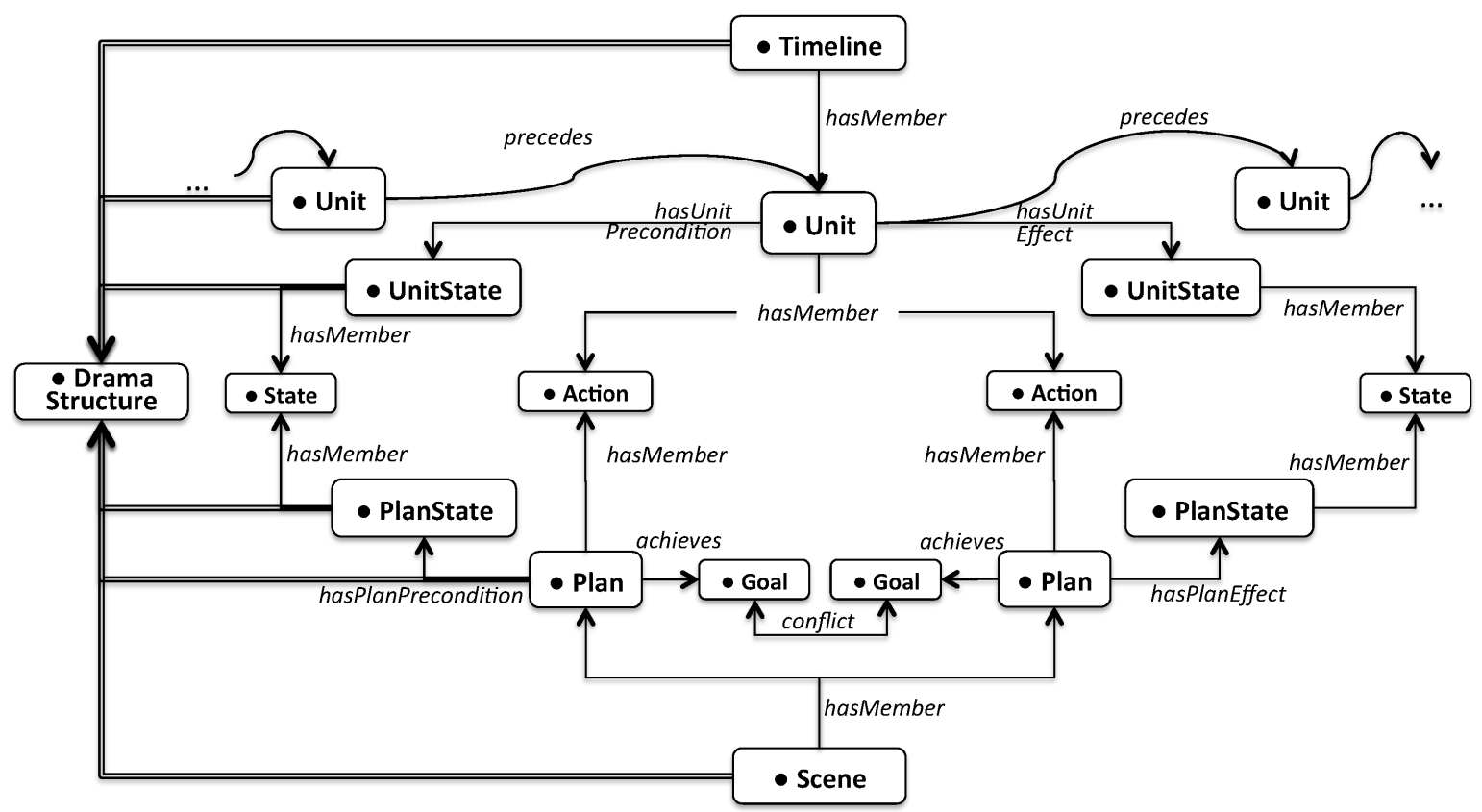

Fig. 4. The drama structures in Drammar: plans, units, planstates, unitstates, timeline, scenes. Subclass relations are represented with a double-line arrow. White boxes are classes; no instances of classes in this figure.

the unit (connected through the property hasUnitEffect) contains the states that hold in the story world after the incidents in the unit occur. The incidents (i.e. the actions) that occur in a unit, as well as the states in the two unit states associated, result from the projection of the actions and states, respectively contained in some plan that constitutes the motivation for the incidents. Two plans that achieve goals in conflict are contained in one scene (class Scene).

For example, the action in the "nunnery" scene is dramatic because of the two characters' conflicting goals: Hamlet wants to turn Ophelia away from the court's influence; Ophelia wants to respect Polonius' authority.

The notion of conflict in Drammar is represented by the opposition of two characters' goals (Figure 4), through the object property labeled as conflict. This object property links two goals that are achieved by two plans that belong to the same scene (an instance of the class Scene).

\section{DRAMMAR AND THE DOCUMENTATION OF CULTURAL HERITAGE}

The representation of drama as intangible cultural heritage provided by Drammar can be seen as part of a larger effort, started approximately two decades ago, to devise formal models for addressing practical concerns of various type in Cultural Heritage, from the management of bibliographical data to the interoperability of cataloging activities. In particular, two models are relevant, namely the Functional Requirements for Bibliographical Entities, known as FRBR [O' Neill, E. T. 2002], and the Conceptual Reference Model (CRM) of cultural heritage, issued by the CIDOC working group of the International Council of Museums (ICOM) [Doerr 2003]. These two models have been recently merged into a common model as a result of a decennial harmonization initiative [Doerr and LeBoeuf 2007]. 
CIDOC CRM has been a pioneering attempt to describe the cultural heritage artifacts by referring not only the artworks themselves, but also to real world entities that are relevant to the art domain and to the functioning of museums in particular. CIDOC CRM is intended as a "common language for domain experts and implementers", specifically aimed at the design of information systems in the field of cultural heritage [Doerr 2003]. Developed along more than two decades, CIDOC CRM is currently released in a semantic format that supersedes the previous conceptual model encoded in the relational data model. Along with the features of clarity and explicitness that characterize semantic representation, a practical advantage of using a semantic format is that, following to the paradigm of Linked Open Data [Bizer et al. 2009], the semantically encoded metadata can be straightforwardly published and shared on the Web.

FRBR, designed with the goal of capturing "the underlying semantics of bibliographic information", acknowledges four main entities: Work, i.e., abstract ideation, Expression, i.e., the encoding of the Work in a specific language (such as text or music), Manifestation, i.e., the embodiment of the Expression in a concrete representation, and Item, a single instance of the Manifestation. Thanks to its capability of dealing with the distinction between the abstract notion of work and its derived entities, FRBR has seen several attempts at applying it to specific examples of cultural heritage, ranging from music [Riley 2008] and performance [Doty 2013] to intangible cultural heritage [Yann 2005]. In particular, [Doty 2013] resorts to FRBR to account for the problem of variation in performance, an acknowledged area of ICH: "the problem of variation is the problem of how, if a Work is defined by all the examples of it, we can determine that two examples that are not identical are nonetheless part of the same Work. This problem is especially pronounced in live performance, which, by its very nature, has the potential for each of its examples to be unique" [Doty 2013] [Fischer-Lichte 2008]. According to Doty, an ontology of drama performance should include the notion of production in order to guarantee the recognizability of a performance with respect the production it belongs to. Although Doty's claim on production is well motivated, here we do not take any position about how the notion of performance can be accommodated into the FRBR model, since the annotation provided by Drammar addresses only, in FRBR terms, the Expression of the play. The description of the drama content provided by Drammar is itself conceptually situated at the level of Expression in FRBR, i.e. an abstract linguistic entity encoded in a Semantic Web language, the Ontology Web Language (OWL), that can be subsequently turned in a specific format among those encompassed by the specifications the version of OWL employed for Drammar (OWL2, see previous section) and finally transferred into a digital resource. So, a play and a specific production of the play can both be separately encoded in Drammar but the representation provided by Drammar does not provide any means to describe the relation between the two, and relies on external models (such as FRBR and its derivatives, including Doty's) to account for this relationship.

Recently, the FRBR model has been challenged by [Renear and Dubin 2007], who points out the inadequacy of the notion of type to describe the transition from Expression to Manifestation and Item in FRBR, and proposes to replace it with the more flexible notion of role. Renear's main argument is that the entities in the Expression-Manifestation-Item triad are not related to the each other by an immutable necessity, but only as the result of a social process of meaning assignment of which linguistic rules are a mere enabling condition. Renear's revision of FRBR, however, does not affect the practical orientation of FRBR, as the author admittedly notices: for practical purposes, in fact, including the preservation of drama as ICH assumed by Drammar, the properties of FRBR entities can be considered fixed and their relationships taken for granted. Encoding the meaning of drama through formal ontologies, then, is in line with Renear' most recent work on preservation: [Sacchi et al. 2011] proposed a model of digital preservation that relies on the distinction between propositional content and symbol structure, and on the mapping between the two. The use ontologies to represent drama con- 
tent is in line with this model, since they provide a powerful and formalized language for transmitting unambiguously a given propositional content across different encoding formats and supports.

Situated at a different conceptual level than Drammar, CIDOC-CRM provides a representation of cultural heritage objects that is functional to the management and planning of the processes that are relevant to their acquisition, custody, preservation, etc. Since Drammar does not represent the production and management metadata of dramatic works, it assumes the framework provided by CIDOCCRM without overlapping with it. For example, a movie may be represented in CIDOC as a conceptual entity having a Title, resulted from a Creation activity and encoded as a Symbolic Object independent of a physical carrier, but the story told by the movie is not within the scope of CIDOC-CRM. Mainly devoted to representing concepts such as ownership, custody and appellation, relevant to the functioning of museum institutions, CIDOC-CRM does not deal with the representation of the content of cultural heritage objects. In the terms of CIDOC-CRM, the digital objects describing a drama item, encoded in Drammar, can be seen as instances of the Information Object class. Subclass of the Propositional Object and Symbolic Object classes, this class which may include instances of a movie or a set of equations as well, can accommodate also graph-like structures, as ontology based representations typically are. The physical counterpart of the abstract encoding, then, would be described as an instance of Physical Man Made Thing, i.e., a physical items generated by human activity.

\section{WORKFLOW FOR BUILDING A DIGITAL ITEM OF DRAMA}

The workflow in Drammar is incremental, thus the consistency of the digital item can be tested at any moment through a visualization system (Section 6). As the construction proceeds, more and more sophisticate structures augment the timeline of incidents extracted from the published text. The item can be revised subsequently, as more knowledge on the drama is available. Figure 5 illustrates the phases of the workflow for constructing the digital item.

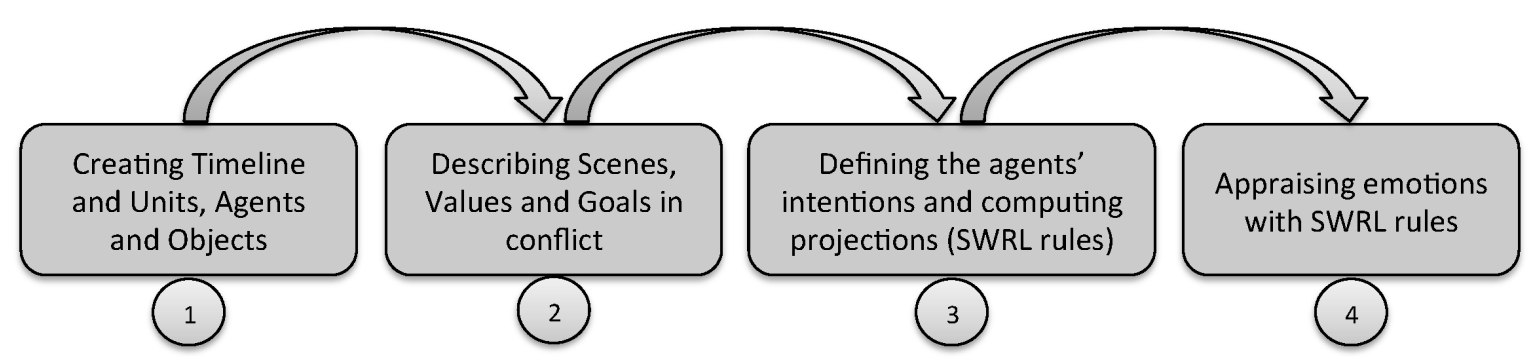

Fig. 5. Workflow for building the digital item for a drama.

\section{Creating Timeline and Units, Agents and Objects}

The construction starts from the encoding of the timeline of incidents (actions) as a sequence of Unit instances (Figure 6). Here, we identify the unit boundaries ${ }^{7}$ and the major actions that occur in them, described through an informal description (e.g. "Hamlet tests Ophelia for honesty and she lies"). In this phase, we also identify the major objects and agents that participate to the incidents.

\section{Describing Scenes and Goals in conflict}

\footnotetext{
${ }^{7}$ An experiment has shown the feasibility of such an approach, see [Lombardo and Damiano 2012], without much discrepancy over different annotators.
} 


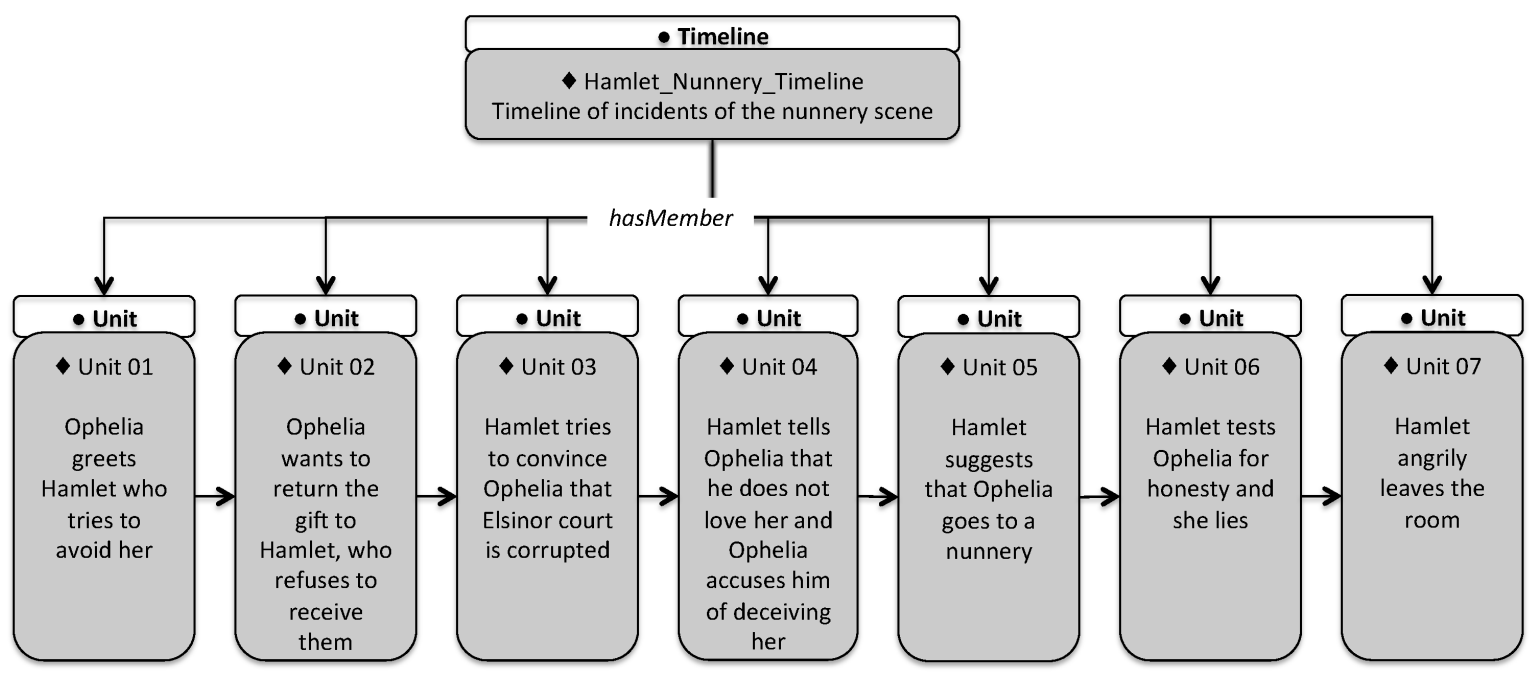

Fig. 6. Creation of the timeline and the units. In these exemplified steps of the workflow application we only show the textual free description of the instances (see the snippets above, for the free descriprions).

Once the sequence of units is defined, we refine the description of the agents involved by explicitly marking the conflicts as well as refining the interplay of the agents. This leads also to identifying the scenes that cluster conflicts together (see Figure 7). Therefore, at this step of the workflow, the units begin to be augmented with goals (e.g., "Hamlet wants to test Ophelia honesty") and values engaged (e.g., "Hamlet Honesty at Stake"). Such values engaged, put at stake, underlie the formation of goals as well as the devise of plans to achieve them.

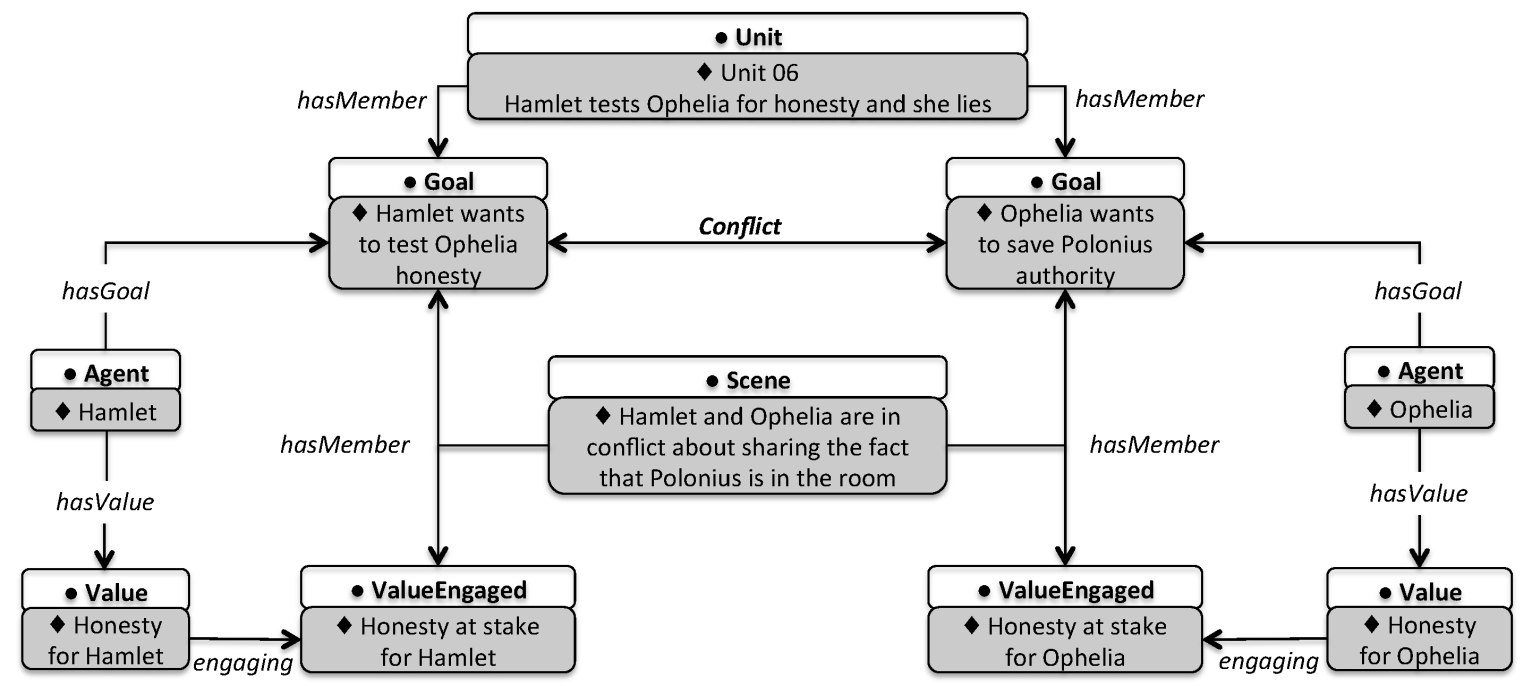

Fig. 7. Agents' goals, values and conflicts for a unit in the "nunnery" scene. 


\section{Defining the intentions}

Then, the deliberative processes underlying the scene. We first identify the simplest plans that motivate the incidents occurring in the units. For each agent, we build base plans (for example, see in Figure 8 the plan "Hamlet intends to ask Ophelia about Polonius' location"). This plan includes the action ("Hamlet asks Ophelia about Polonius location"), and has precondition and effect states. In the "nunnery" scene, this plan is a subplan of "Hamlet intends to test Ophelia for honesty". In principle this subplan is followed by another subplan that is not deployed because Ophelia's answer is not what Hamlet was expecting (she lies about Polonius' location). Then, we proceed with the description of the incidents, built formally through the predicates and roles determined by the linguistic frames described in Section 3.1.

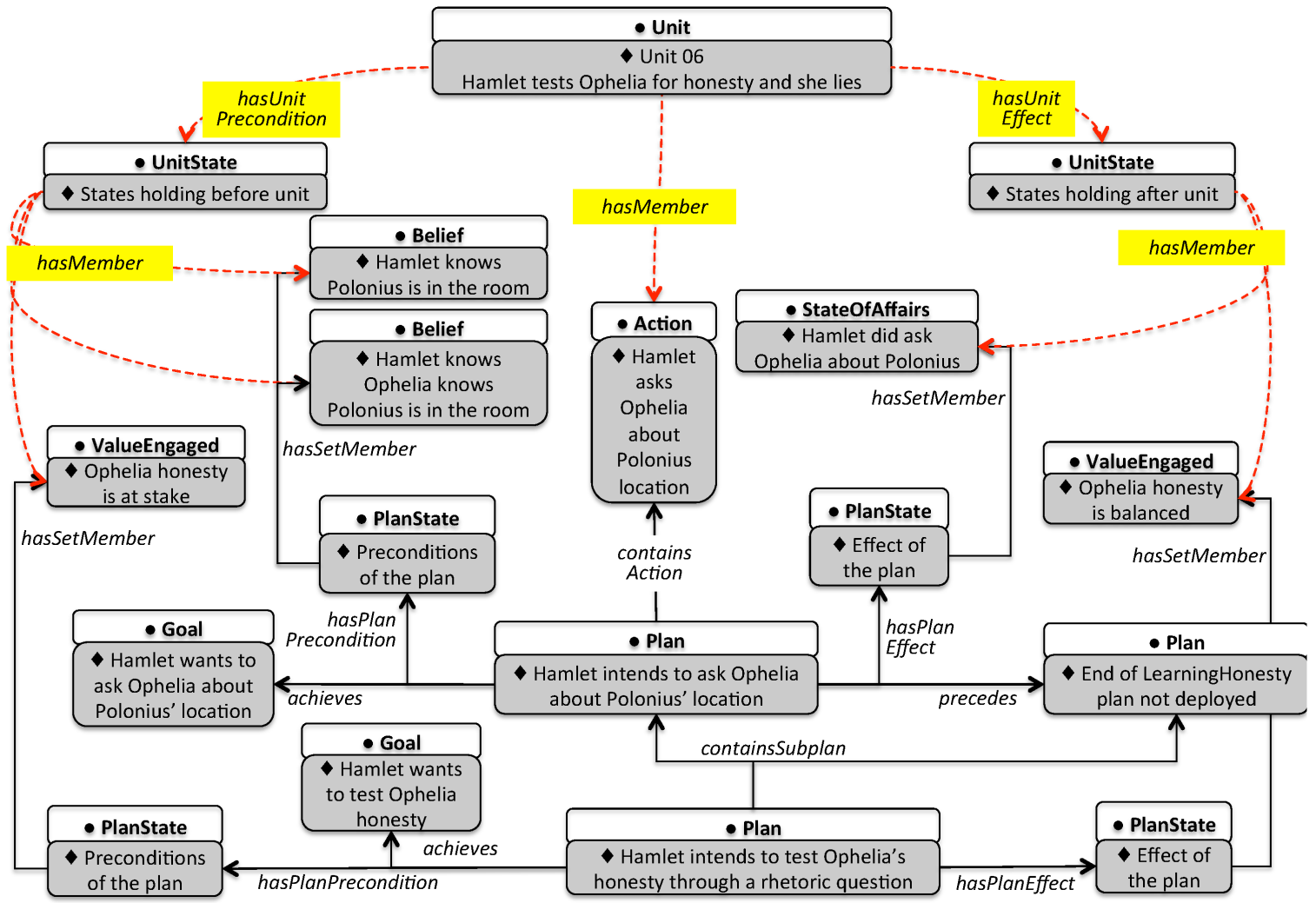

Fig. 8. Plans and actions (relations in solid lines), with the augmentations provided by rule execution (relations with dashed lines).

Finally, once we have associated plans and units, SWRL rules project the actions described formally in the plans onto the units in the timeline, to match the incidents (dashed lines in Figure 8). This leads to the creation, before and after each units, of precondition and effect states projected from the preconditions and effects of the plans (of several agents) that span the unit. Incrementally, we can continue to add agents' plans, aimed at enriching the plan structure, and run the reasoning modules to maintain the coherence. 
Appraising emotions with SWRL rules

Values put at stake as a result of some plan accomplishment and goals in conflict are the input to SWRL rules for the emotion appraisal. These rules compute the emotions felt by some agent given two main elements, namely the values of the same agent put at stake (or re-balanced) and the achievement of her/his own goals with respect to other agents' conflicting goals.

Figure 9 shows the input individuals and relations that feed three rules for the appraisal of Distress, Reproach and Anger respectively, as well as the results of the rule activation. In particular, Hamlet feels Distress about his value honesty put at stake by the achievement of Ophelia's plan that is to save Polonius' authority through lying. Hamlet also feels Reproach for Ophelia because his goals of proving Ophelia's honesty fails while Ophelia's goal to save Polonius' authority is achieved and the two goals are in conflict. Finally, the combination of Distress and Reproach, according OCC theory, causes Hamlet to feel anger toward Ophelia.

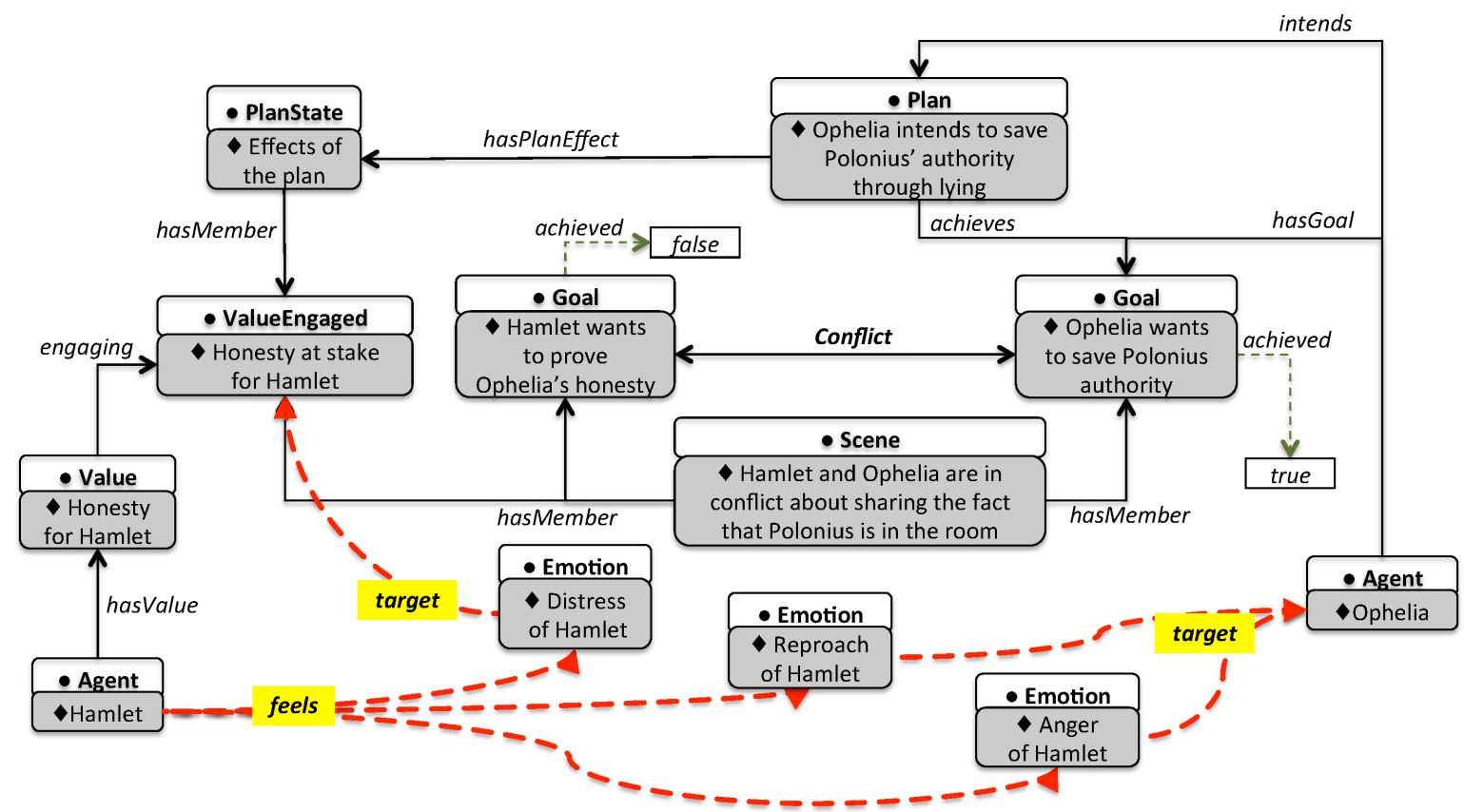

Fig. 9. Concepts and relations that trigger the SWRL rules that calculate Hamlet's emotions in the "nunnery" scene. Solid lines represent what is manually annotated, dashed lines represent relations that are calculated automatically by the rules.

\section{TESTING THE DRAMMAR ONTOLOGY}

This section tests the application of the Drammar ontology in two concerns: the first is whether the ontology is usable for practitioners; the second is the encoding into Drammar of two well known theories of drama, namely Freytag's and Polti's theories [Freytag 2004] [Polti 1895]. 


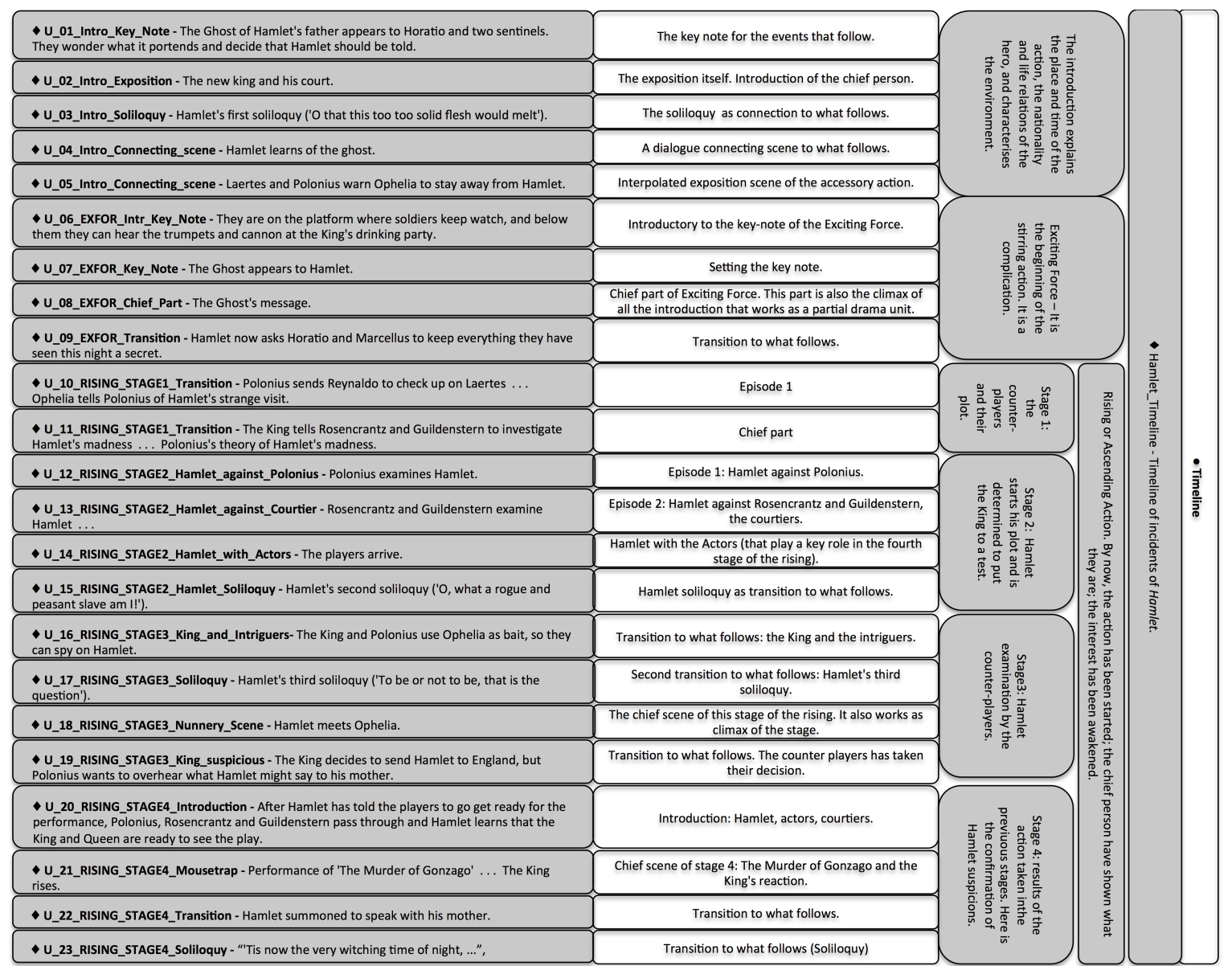

Fig. 10. Visual schema of how Drammar encodes a segment of Freytag's partition of Hamlet. On the left, the list of narrative units listed by Freytag and encoded in Drammar. In the middle (white boxes), Freytag's description of the narrative function of each unit. On the right, the first three parts of Freytag's triangle: Introduction, Exiting Force, and Rising (note that the Rising is further divided in four stages), also encoded in Drammar as macro units.

\subsection{Enconding drama theories in Drammar}

In this section, we provide a testing of the expressiveness of Drammar through its capability of encoding two different approaches to the description of drama. Drammar was built by identifying the elements that are common to many theories of drama and providing a representation structure that allows the verification of the consistency of representations. The goal of Drammar is to be compliant with the major theories and so to provide a form of normalization for some theories that allows to share a common ground of representation.

Along centuries, drama scholars have produced a large amount of interpretations, but they mostly accounted for the philological references or for the latent meaning of the work (see, for example, [Bloom 1998] and [Kott 1974]). Drammar does not take into account these theories, because they are concerned 
with the historical relevance of the work rather than with its structural elements. Even though, among these approaches it is common to find differences concerning the cultural appraisal of the work. For example, it is possible to describe how Shakespeare's dramas have been received in XVIII century France and how it has been "rewritten" to fit neoclassical rules, as in the case of Hamlet: tragédie, imitée de l'anglois by Jean-François Ducis in 1770 [Pemble 2005]. Or, it is common to argue about the underlying meaning of the text, whether it is a real revenge drama or is a sort of psychological struggle of a young intellectual [Singh 2013].

Drammar is not designed to help to decide whether Hamlet is political or existential. Although it is easy to find such type of analysis that are overtly in contrast among them, they are usually compared by means of philological or cultural studies. Yet, there is a tradition of drama critics addressing specific structural elements of the work, and shedding light on the drama from different points of view. This is the case we address here to test the expressiveness of Drammar. We provide an encoding of two major theories. Freytag's theory deals with the major phases of narration in drama [Freytag 2004]. Freytag has devised his famous triangle thus suggesting a five-act structure (still famous nowadays in film, TV series, and advertisement production) recently pointed out as the major factor of the success of advertisement and marketing [Herman et al. 2010]. Polti's theory addresses the type of contents in drama, hence he is concerned with the kind of situations that can be perceived as dramatic [Polti 1895]. His analysis of a very large collection of dramas has allowed him to classify 36 dramatic situations, currently influential in game design and interactive storytelling [Hall and Baird 2008].

Figure 10 displays Freytag's account of Hamlet, as encoded with Drammar. Freytag lists the incidents of the story regardless of the division into scene and acts. He prefers to break the plot into phases corresponding to the elements of his arc (Introduction, Exciting Force, Rising, etc.). Drammar is able to represent the recursive structure of the units and macro units (in grey in the figure) and to sequence them into a timeline. Given the motivational bias of our ontology, Drammar is suited to provide a straightforward representation of the units with a definite actional content (e.g. unit 19, "The King decides to send Hamlet to England"), because they can be described as a instances of the class Action. Drammar is not immediately effective to represent the narratological function of the units (e.g. unit_02_Intro_Exposition "The new king and his court"), because the ontology does not contain classes designed to represent the phases of the narrative (e.g., introduction, exposition, etc.). These units can rather be represented as actions, neglecting their non dramatic function, which may not fit well in the interplay of intentions and actions that form the structure of drama.

The motivational bias of our ontology, on the other hand, it is more effective to represent a character based approach to drama, as the one proposed by Polti's theory. Figure 11 illustrates how the classes, properties and attributes of Drammar can encode Polti's description of the 4th situation named "Vengeance taken for kin upon kin", that apply to Hamlet. For this situation, the dynamics elements described by Polti are: a Guilty Kinsman; an Avenging Kinsman; a remembrance of the Victim relative of both. The first two are put into conflict over wrongdoing to the Victim. Drammar is able to represent the three dynamics element as Agents with Goals. The goals have a propositional content (Goal Schema) and, leveraging on external resources (Framenet), it is possible to describe the content of the situation in terms of roles and attributes (e.g. Hamlet plays the role of the avenger, while Claudius is the offender). Furthermore, Drammar can encode the core dramatic feature describing the conflict among Hamlet and Claudius (in the figure, the Conflict property between the the instances of the class Goal).

Because of Drammar account for both the listing of units and description of agents' motivations, we believe it helps to encode a more comprehensive representation of drama as ICH, thus providing scholars with a new form of accessing the work through a shared formal expression (as in the case of the scene described in the following Section 6.2 and Figure 12). 


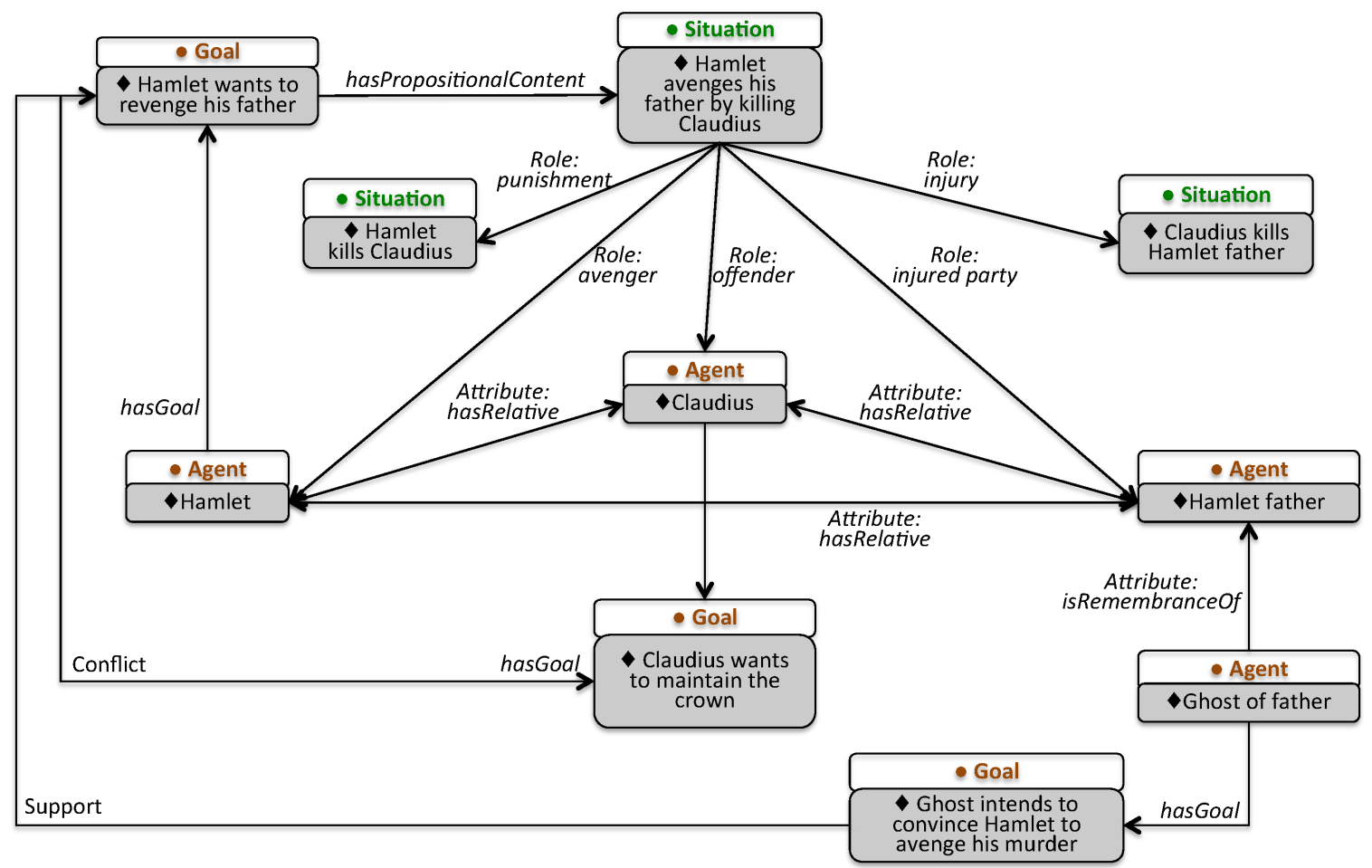

Fig. 11. Normalization of Polti's characterization of Hamlet in Drammar terms. Notice that frames and roles have been collapsed to edge labels for simplicity of display.

\subsection{Usability of Drammar}

In this section, we describe the results of a focus group we conducted with practitioners and scholars of drama, where we presented the major tenets of the ontological representation together with a simplified visualization in order to test the effectiveness of the representation and the usability of the interface. Here we proceed by quickly illustrating the visualization system and then the results of the focus group.

Though the ontological representation allows for a semantics-based machine-readable encoding format of intangible heritage, the access to such knowledge is restricted to computer scientists in the OWL language. Even graphic interfaces, such as the ones in the figures above, are very complex and intricate, in the absence of the capability of selecting specific concepts and relations to display and of an appropriate layout that highlights selected features. The introduction of a visualization method is necessary to support the use by scholars and enthusiasts, avoiding the direct use of the formal ontological encoding. The visualization system we employed in the focus group displays the alignment of the agents' plans and goals with the actions of the timeline. Details of the design and implementation can be found in [Lombardo and Pizzo 2015].

Figure 12 shows the overall visualization of the "nunnery" scene of Hamlet. Four agents are involved in the "nunnery" scene (Hamlet, Ophelia, Polonius, Claudius) marked by different colors; however, this scene is driven by Hamlet and Ophelia's plans: the intentions of Polonius and Claudius, who organized 
Ophelia's meeting, are situated at a higher level and can be visualized in a wider context. The area called "Timeline", at the top of the figure, consists of: (1) the actual incidents (in green background and white text); (2) the precondition and effect states projected from plans (in red background and white text); (3) non-executed actions and non-reached states projected from plans that failed (grey cells with no text).

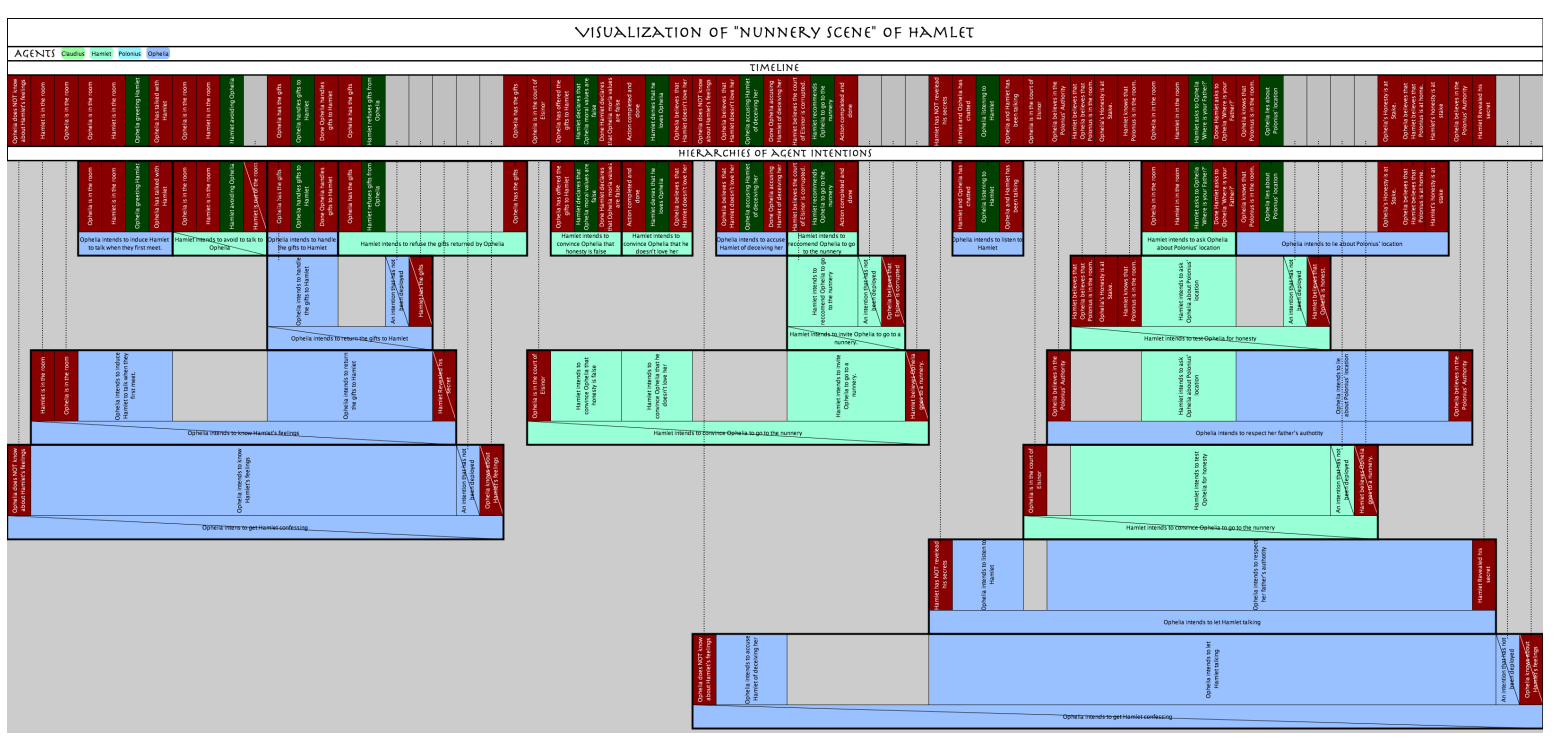

Fig. 12. Overall visualization of the "nunnery" scene (original $3250 \times 1512$ pixels). From the top: the timeline (colored boxes are actions and states projected by the plans, large boxes below; grey boxes are actions and states of the intentions that have not been realized); the hierarchy of plans, with different color for each character (larger, lower plans include smaller, higher plans as subplans). Though the content of the cells is not legible in this figure, it provides an overview of the contribution of the characters' intentions to incident plot of the scene (see text).

In the area called "Hierarchy of Agents' Intentions", the colors identify the agents who intend the plans visualized in the schema. Each plan is visualized as a horizontal rectangle, with actions, subplans and precondition and effect states laid out as daughters; each subplan is aligned with the plan that rewrites it, until the basic actions and states aligned with elements in the timeline. Dominance relations are represented by different layers: plans closer to the timeline consist of a single action bordered by precondition and effect states, on the left and on the right, respectively; higher plans in the hierarchy (lower in the figure) consist of a sequence of subplans bordered again by precondition and effect states. All actions and states are projected onto the timeline (the dashed lines in the figure). Each action/incident or state is represented by a vertical cell; in this area of the visualization, we also show the elements that are not realized in the timeline (i.e. plans not achieved and states that do not hold) barred with a diagonal line. These representations have been designed of large sizes for printing and contain large text for better visualization.

In the visual representation, the user can appraise:

- the vertical alignment between the agents' plans and the actions that occur in the timeline, following the vertical dashed lines;

- the story advancements through the states that hold along the timeline axis;

ACM Journal on Computing and Cultural Heritage, Vol. 2, No. 3, Article 1, Publication date: May 2010. 
- the evolution of the behavior of a single agent, by tracing the sequence of plans of the same color on the horizontal axis;

-what elements (the grey cells) predicted by the plans are missing from the timeline because of plan failures (horizontal cells with a bar);

-what actions in the timeline are not aligned to any element in the hierarchy, meaning that some scholar interpretation is incomplete;

- the depth of the hierarchy for a single agent in some portion of the timeline, distinguishing the actions pertaining to some reacting behavior (little depth) from the actions that result from a longterm planned behavior (great depth);

- the orchestration of conflicts through the vertical alignment of plans of different agents, mixing successes and failures;

Given such a visualization system, we conducted a focus group with drama experts to assess the interest of scholars and practitioners in the visualization of drama and the immediacy of the latter. A group of 5 subjects was given a demo of the visualization prototype, followed by a discussion of its possible advantages for their work. The participants were scholars in film and theatre studies, chosen for their experience in production and acting. During the discussion, there was a substantial agreement on the usefulness of computer-based tools for the study and analysis of drama, but a scarce awareness about the use of digital formats in cultural heritage, and of semantic encoding in particular.

After the demo, the participants were asked to answer an anonymous questionnaire of 15 questions. Designed with the goal of relating the interest in the visualization with the acceptance of the encoding conducted with Drammar, the questionnaire was distributed online and included three main groups of questions. The first group of questions (questions 1 to 4 ) specifically addressed the research interests of the participant and her/his professional experience about drama. About the research interests and the professional profiles of the participants, their research resulted to be mainly focussed on film and theatre studies (Question 1, free text), with a specific interest in acting mentioned by 2 participants, and a focus on theatre and on cinema respectively for 2 participants (Question 2, free text). The courses taught by the participants ranged from Digital animation to Film studies and Theatre studies (Question 3), and their computer literacy was declared as basic (use of office automation tools) by all of them (Question 4).

The second group of questions (questions 5 to 7) addressed the suitability of the categories implied by Drammar for the analysis of drama. The participants' opinion about Drammar expressed a substantial agreement on its suitability to describe drama. 4 participants out of 5 answered "mostly adequate" to the question about the adequacy of Drammar to the representation task (Question 5, the possible answers were "No adequate at all", "Partly adequate", "Acceptably Adequate", "Mostly adequate", "Adequate", one participant did not provide any answer). The meaning of the single categories (Question $6^{8}$ ) was deemed clear, for both structural categories (e.g. Timeline) and intention-oriented categories (e.g. "Plan" and "Goal"). The participants did not request to add further categories to drama representation (Question 7, free comment).

The third group of questions (questions 8 to 15) specifically addressed the proposed visualization. Question 8 (again, a single participant did not answer) addressed the acceptance of the visualization, with an average score (on a scale from 1 to 5 ) of 3.5 for "effectiveness", 2.75 for "clarity" and 2.75 for "intuitiveness", thus pointing out the still incomplete design of the visualization tool and the need for a substantial training for the users to decode it, but also stating clearly its potential effectiveness for the ${ }_{8}^{\mathrm{A} \text { checklist }}$ was provided including the following categories: Character, Goal, Plan, Plan/Subplan relation, Action, Emotion,
Value, Belief, Augmented timeline, Other. 
task at hand. Concerning the purpose of using the visualization tool (Question $9 a$, yes/no question), 3 participants out of 4 answered that they would like to use the tool for teaching purposes, but only 2 out of 4 answer they would like to use it for research purposes (Question $9 b$, yes/no question). As for the use of the visualization (Question 10, free comment), the type of work envisaged were films (1 participants) and theatre plays (2 participants). The participants did not identify any elements that they would like to include or expunge from the visualization (Questions 11 and 12, both presented as a checklist of visualized elements), consistently with the previously expressed agreement on the categories of Drammar. Given their previous experience (Question 13, free comment) as actors, directors and scholars, the possible uses envisaged by the participants for the visualization were the following: helping the students in the analysis of the text, and helping the director and the scenographer to identify and manage the element of the mise-en-scène. Additional comments (Question 15) mainly concerned the visual appearance of the visualization interface, with sparse observations about the inadequacy of the colors, layout, etc. A single participant addressed the gap between the accuracy and completeness of the annotation with the limited selection of the visualized elements.

\section{RELATED WORK: ANNOTATION, ONTOLOGIES, VISUALIZATION}

This section addresses the languages and tools for the annotation of drama and for the visualization of dramatic qualities, that have some (even loose) relation to our work.

Annotation of cultural heritage The problem of the annotation of tangible cultural heritage items has received some attention recently. Some authors have resorted to Wikipedia, which offers in-depth descriptions and links to related articles, and is thus a natural target for the automatic enrichment of cultural heritage items (see, e.g., [Agirre et al. 2012]). In the specific domain of the performing arts (which are related to drama), the tool MyStoryPlayer is a specifically designed interface for the semantic annotation of documents (such as video, audio, text, image, etc.) in RDF format and the navigation of the annotations according to one's own non-linear experience or path [Bellini et al. 2011] [Bellini et al. 2014].

An project that can be related to the annotation of ICH is the Motion Bank initiative carried on by the Forsythe Company. The project focus is to create a repository of online digital scores for choreographies, produced in collaboration with guest artists, that can be useful in research and teaching and to involve collaborative dance practices. The language employed in this initiative refers to the "synchronous object approach", which examines choreographies through the principle of counterpoint, i.e. "intermittent and irregular coincidence of attributes between organizational elements [that] produces an ordered interplay"'. The structural elements taken into account are: material movements (a set of 25 choreographic themes), a cueing system (aural or visual signals that triggers events) that determines the flow of the dance, alignments (short instances of synchronization between dancers that share some action attributes).

Annotation and visualization of drama In the domain of annotation for drama specifically, there exist approaches that guide the annotation for the formal encoding of the story elements. The Story Intention Graph [Elson 2012] relies on the representation of the short-term characters' intentions to build an interpretive layer of a narrative text; however, such an approach lacks the expressive power to represent long term goals of the characters (which we encode in the hierarchy of plans) and only focus on immediate goal-plan relations (what we call base plans). The Stories Ontology ${ }^{10}$, developed in collaboration with BBC for the application to news, fiction (the storylines of Doctor Who

\footnotetext{
${ }^{9} \mathrm{http} / / /$ synchronousobjects.osu.edu/assets/objects/introduction/danceDataObjectEssays.png

${ }^{10} \mathrm{http}: / /$ www.contextus.net/stories
}

ACM Journal on Computing and Cultural Heritage, Vol. 2, No. 3, Article 1, Publication date: May 2010. 
episodes), and historical facts, is an event-(instead of character-) based description of the timeline of story incidents, with no interpretive intents. Mainly inspired by a production support approach and oriented to trans-media comparisons, it lacks the expressive power to catch all the dramatic elements surveyed in Section 3.

The visualization of story relations has attracted the attention of visual artists and amateurs to provide unique maps for orientation. This is particularly useful for stories with intricate plots that are not immediate to grasp (see, e.g., the visualization of two Nolan's films Memento ${ }^{11}, 2000$, and Inception ${ }^{12}$, 2010), but also to trace the overall involvement of characters, visualized as horizontal chronological lines that converge and diverge, illustrating their mutual interactions as well as their relationship in time with places and/or collective events (see, e.g., the movie narrative charts ${ }^{13}$. The latter visual design was then automatized through some algorithmic approaches in [Tanahashi and Ma 2012] and [Liu et al. 2013]. On a more productive side, a number of visual interfaces are provided with software tools that have been developed to assist the creation and production of dramas. For example, the writing assistant Dramatica Pro ${ }^{14}$ visualizes the building blocks of a plot structure, with diagrams for plot progression and story points, that helps the writer in controlling and balancing the tension within the story development. The connections fleshed out are useful to connect the several professionals of the production, while leaving unexplored the possibilities of addressing the more motivational features of the drama.

Ontologies and cultural heritage The use of ontologies is not new in cultural heritage documentation and promotion. Since Berners Lee's manifesto of Semantic Web appeared in 2001 [Berners-Lee et al. 2001], semantic technologies have proven their suitability for the dissemination of cultural heritage [Doerr 2003]. Several research projects have explored the application of semantic technologies to cultural heritage. One of the most representative, however, is the Finnish Culture Sampo project [Hyvönen et al. 2009]: conceived of as a large-scale demonstrator, Culture Sampo is a cultural heritage portal entirely relying on a "mash up" of domain ontologies that encode all the relevant features of artworks, from geographical data to craftsmanship and content.

The semantic annotation of media is also one of the main applications of ontologies, aimed a reducing the semantic gap between the data they contain and the content encoded in these data. Since drama is often conveyed through media (from films and performance recordings to radio drama), representing the multimedia data and processes is a necessary step to cope with the semantic gap. Ontologies of multimedia, however, do not directly address the content level, since they describe the procedures for encoding and manipulating multimedia data. COMM [Arndt et al. 2007], in particular, provides the conceptual toolkit for the description of multimedia data from a structural viewpoint: it describes, for example, the notion of "spatial region" in images, or the tasks conducted on image data, such as their encoding in a specific format through an algorithm or their annotation with metadata. In the semantic annotation of media, these ontologies can be employed to describe the encoding of data, implied by the notions of Expression and Manifestation in FRBR.

Finally, the relation of Drammar with the vocabularies of semantic descriptors employed for media annotations is more complex. Multimedia vocabularies, such as TRECVID [Naphade et al. 2006] or the more sophisticated VERL ontology [François et al. 2005], are usually coupled with low level representations of multimedia data (typically, video) for indexing their content so as to make it searchable by access tools. However, the features they address, such as the occurrence of basic events in the audio-

\footnotetext{
${ }^{11} \mathrm{http}: / /$ visual.ly/memento-scene-timeline, visited December 2014

${ }^{12} \mathrm{http}: / /$ visual.ly/inception-timeline-visualisation, visited December 2014

${ }^{13} \mathrm{http}$ ///store.xkcd.com/collections/posters/products/movie-narrative-charts-poster, visited December 2014).

${ }^{14} \mathrm{http}: / /$ www.writersstore.com/dramatica-pro-story-development-software/,visitedDecember2014.
} 
visual stream and/or the appearance of physical entities, are situated at a lower level than Drammar, which provides a meaning level (i.e., dramatic structures) that is conceptually more abstract. Semantic descriptors of entities and events, however, may be coupled with the description of incidents provided by Drammar, with the goal of anchoring the annotation of drama into multimedia data (a precursor of this was encompassed by the CADMOS project [Lombardo and Pizzo 2014]).

\section{CONCLUSION}

This paper has introduced the drama as a form of intangible cultural heritage. We have identified a set of dramatic elements that are distinctive of the (tangible) dramatic media items. We have presented a solution for the preservation of those elements in terms of a formal encoding of metadata through a computational ontology. Then, compliant with the methodology carried out by initiatives such as CIDOC-CRM for representing explicitly the conceptual model underlying the description of cultural heritage artifacts, we have proposed a semantic representation of the conceptual model of drama. By leveraging the semantic description, several advantages are obtained beyond the benefits of a clear, unambiguous description of the model. The semantic format itself is more interoperable than traditional relational models, which depend on implementation, and complies with the paradigm of linked data, through which data can be published on the web and made available to joint research efforts and dissemination. Moreover, it may be integrated in text annotation by keeping the textual and the conceptual level aligned, yet distinct.

We have also provided a workflow for encoding a digital item for drama and we have tested the suitability of the approach through the encoding of two well known theories of drama and with the participation of drama scholars and practitioners. A method for the visualization of the drama encoding has turned out to be useful in teaching and production and promising to create collections of encoded drama items. The formal encoding can trigger further research in representation and analysis of drama and can constitute the base for effective learning objects in the didactics of drama studies.

As future work, the formal encoding of drama, accompanied by the visualization interface, will be applied to a number of tasks: in production, to support authors, directors and producers in keeping under control the process of mise-en-scène; in the search and retrieval of media items, through the encoded dramatic features; in the editing of dramatic fragments for specific tasks, such as summarization and trailer making. The next steps will be the engagement of practitioners who can point out specific issues to be addressed for making the representation more and more useful in real tasks with dramatic media, bridging the gap between the potential of formal models and the awareness of digital formats by drama scholars.

\section{REFERENCES}

2003. Convention for the Safeguarding of the Intangible Cultural Heritage Paris, 17 October 2003 http://www.unesco.org/culture/ich/en/convention. (October 2003). http://www.unesco.org/culture/ich/en/convention

Eneko Agirre, Ander Barrena, Oier Lopez de Lacalle, Aitor Soroa, Samuel Fernando, and Mark Stevenson. 2012. Matching Cultural Heritage items to Wikipedia. In Proceedings of the Eight International Conference on Language Resources and Evaluation (LREC'12) (23-25), Nicoletta Calzolari (Conference Chair), Khalid Choukri, Thierry Declerck, Mehmet Uğur Doğan, Bente Maegaard, Joseph Mariani, Jan Odijk, and Stelios Piperidis (Eds.). European Language Resources Association (ELRA), Istanbul, Turkey.

Aristotle. 2008. Poetica. Einaudi, Turin, Italy.

Aristotle. 2013. Poetics. Oxford University Press.

Richard Arndt, Raphaël Troncy, Steffen Staab, Lynda Hardman, and Miroslav Vacura. 2007. COMM: Designing a WellFounded Multimedia Ontology for the Web. In The Semantic Web, Karl Aberer, Key-Sun Choi, Natasha Noy, Dean Allemang, Kyung-Il Lee, Lyndon Nixon, Jennifer Golbeck, Peter Mika, Diana Maynard, Riichiro Mizoguchi, Guus Schreiber, and Philippe Cudré-Mauroux (Eds.). Lecture Notes in Computer Science, Vol. 4825. Springer Berlin Heidelberg, 30-43. DOI : http://dx.doi.org/10.1007/978-3-540-76298-0_3 
Collin F. Baker, Charles J. Fillmore, and John B. Lowe. 1998. The Berkeley FrameNet Project. In Proceedings of the 36th Annual Meeting of the Association for Computational Linguistics and 17th International Conference on Computational Linguistics - Volume 1 (ACL '98). Association for Computational Linguistics, Stroudsburg, PA, USA, 86-90. DOI : http://dx.doi.org/10.3115/980845.980860

Pierfrancesco Bellini, Paolo Nesi, and Marco Serena. 2011. MyStoryPlayer: Semantic audio Visual Annotation and Navigation Tool. In The 17th International Conference on Distributed Multimedia Systems. Florence. http://www.ksi.edu/seke/Proceedings/ dms11/DMS/23\_Pierfrancesco \_Bellini.pdf

Pierfrancesco Bellini, Paolo Nesi, and Marco Serena. 2014. MyStoryPlayer: experiencing multiple audiovisual content for education and training. Multimedia Tools and Applications (2014), 1-41. DOI:http://dx.doi.org/10.1007/s11042-014-2052-9

Tim Berners-Lee, James Hendler, and Ora Lassila. 2001. The Semantic Web - Computers navigating tomorrow's Web will understand more of what's going on-making it more likely that you'll get what you really want. Scientific American. 284, 5 (2001), 34 .

Christian Bizer, Tom Heath, and Tim Berners-Lee. 2009. Linked Data - The Story So Far. International Journal on Semantic Web and Information Systems 5, 3 (2009), 1-22. DOI: http://dx.doi.org/10.4018/jswis.2009081901

Harold Bloom. 1998. Shakespeare : the invention of the human. Riverhead Books, New York.

Michael E. Bratman. 1987. Intention, Plans, and Practical Reason. Harvard University Press, Cambridge (MA).

Cleanth Brooks and Robert B. Heilmann. 1946. Understanding Drama. G.G. Harrap.

Christie Carson. 1997. Drama and theatre studies in the multimedia age: 'Reviewing the situation'. Literary and Linguistic Computing 12, 4 (1997), 269-275. DOI: http://dx.doi.org/10.1093/llc/12.4.269

Mario Cataldi, Rossana Damiano, Vincenzo Lombardo, and Antonio Pizzo. 2013. Lexical Mediation for Ontology-Based Annotation of Multimedia. In New Trends of Research in Ontologies and Lexical Resources, Alessandro Oltramari, Piek Vossen, Lu Qin, and Eduard Hovy (Eds.). Springer Berlin Heidelberg, 113-134. DOI : http://dx.doi.org/10.1007/978-3-642-31782-8_7

Gerard De Melo, Fabian Suchanek, and Adam Pease. 2008. Integrating YAGO into the Suggested Upper Merged Ontology. In Tools with Artificial Intelligence, 2008. ICTAI'08. 20th IEEE International Conference on, Vol. 1. IEEE, 190-193.

Martin Doerr. 2003. The CIDOC Conceptual Reference Module: An Ontological Approach to Semantic Interoperability of Metadata. (2003). http://www.aaai.org/ojs/index.php/aimagazine/article/view/1720

Martin Doerr and Patrick LeBoeuf. 2007. Modelling Intellectual Processes: The FRBR - CRM Harmonization. In Digital Libraries: Research and Development, Costantino Thanos, Francesca Borri, and Leonardo Candela (Eds.). Lecture Notes in Computer Science, Vol. 4877. Springer Berlin Heidelberg, 114-123. DOI : http://dx.doi.org/10.1007/978-3-540-77088-6_11

Colin Doty. 2013. The Difficulty of An Ontology of Live Performance. InterActions: UCLA Journal of Education and Information Studies 9, 1 (2013). http://www.escholarship.org/uc/item/3jf4g75m

Lajos Egri. 1946. The Art of Dramatic Writing. Simon and Schuster, New York.

Keir Elam. 1980. The Semiotics of Theatre and Drama. Methuen, London and New York.

David K. Elson. 2012. DramaBank: Annotating Agency in Narrative Discourse. In Proceedings of the Eighth International Conference on Language Resources and Evaluation (LREC 2012). Istanbul, Turkey.

Martin Esslin. 1988. The Field of Drama. Methuen, London.

Richard E. Fikes and Nils J. Nilsson. 1971. Strips: A new approach to the application of theorem proving to problem solving. Artificial Intelligence 2, 3-4 (1971), 189 - 208. DOI : http://dx.doi.org/10.1016/0004-3702(71)90010-5

Erika Fischer-Lichte. 2008. The Transformative Power of Performance: A New Aesthetics. Taylor \& Francis. https://books.google. co.uk/books?id=Ocwq3jxiL-8C

Alexandre R.J. François, Ram Nevatia, Jerry Hobbs, and Robert C. Bolles. 2005. VERL: An Ontology Framework for Representing and Annotating Video Events. IEEE MultiMedia 5 (2005), 76-86.

Gustav Freytag. 2004. Technique of the Drama: An Exposition of Dramatic Composition and Art. University Press of the Pacific. Aldo. Gangemi and Valentina Presutti. 2009. Ontology design patterns. Handbook on Ontologies (2009), 221-243.

Nicola Guarino, Daniel Oberle, and Steffen Staab. 2009. What Is an Ontology? In Handbook on Ontologies, Steffen Staab and Rudi Studer (Eds.). Springer Berlin Heidelberg, Berlin, Heidelberg, 1-17. DOI : http://dx.doi.org/10.1007/978-3-540-92673-3

Richard Hall and Kirsty Baird. 2008. Improving Computer Game Narrative Using Polti Ratios. The International Journal of Computer Game Research 8, 1 (September 2008).

Jeffrey Hatcher. 1996. The Art and Craft of Playwriting. Story Press, Cincinnati, Ohio.

T. Heath and C. Bizer. 2011. Linked data: Evolving the web into a global data space. Synthesis Lectures on the Semantic Web: Theory and Technology (2011), 1-136.

David Herman, Manfred Jahn, and Marie Laure Ryan. 2010. Routledge Encyclopedia of Narrative Theory. Taylor \& Francis. https://books.google.it/books?id=oX8hmVw \-yXYC 
Eero Hyvönen, Eetu Mäkelä, Tomi Kauppinen, Olli Alm, Jussi Kurki, Tuukka Ruotsalo, Katri Seppälä, Joeli Takala, Kimmo Puputti, Heini Kuittinen, Kim Viljanen, Jouni Tuominen, Tuomas Palonen, Matias Frosterus, Reetta Sinkkilä, Panu Paakkarinen, Joonas Laitio, and Katariina Nyberg. 2009. CultureSampo: A National Publication System of Cultural Heritage on the Semantic Web 2.0. In The Semantic Web: Research and Applications, Lora Aroyo, Paolo Traverso, Fabio Ciravegna, Philipp Cimiano, Tom Heath, Eero Hyvönen, Riichiro Mizoguchi, Eyal Oren, Marta Sabou, and Elena Simperl (Eds.). Lecture Notes in Computer Science, Vol. 5554. Springer Berlin Heidelberg, 851-856. DOI : http://dx.doi.org/10.1007/978-3-642-02121-3_69

Jan Kott. 1974. Shakespeare Our Contemporary. Norton. https://books.google.co.uk/books?id=QIrdQfCMnfQC

Yves Lavandier. 1994. La dramaturgie. Le clown et l'enfant, Cergy. http://www.clown-enfant.com/leclown/dramaturgie/

Shixia Liu, Yingcai Wu, Enxun Wei, Mengchen Liu, and Yang Liu. 2013. StoryFlow: Tracking the Evolution of Stories. IEEE Transactions on Visualization and Computer Graphics 19, 12 (Dec. 2013), 2436-2445. DOI : http://dx.doi.org/10.1109/TVCG.2013.196

Vincenzo Lombardo, Cristina Battaglino, Antonio Pizzo, Rossana Damiano, and Antonio Lieto. 2014. Coupling conceptual modeling and rules for the annotation of dramatic media. Semantic Web 00 (2014), 1-32. DOI : http://dx.doi.org/10.3233/SW-140156

Vincenzo Lombardo and Rossana Damiano. 2012. Commonsense knowledge for the collection of ground truth data on semantic descriptors. In Proceedings of the 2012 IEEE International Symposium on Multimedia (ISM 2012). IEEE Computer Society, 78-83.

Vincenzo Lombardo and Antonio Pizzo. 2014. Multimedia tool suite for the visualization of drama heritage metadata. Multimedia Tools and Applications (2014), 1-32. DOI : http://dx.doi.org/10.1007/s11042-014-2066-3

Vincenzo Lombardo and Antonio Pizzo. 2015. The visualization of drama hierarchies. In Proceedings of 10th International Joint Conference on Computer Vision, Imaging and Computer Graphics Theory and Applications (VISIGRAPP 2015). 8.

David Mamet. 1998. Three Uses of the Knife: On the Nature and Purpose of Drama. Columbia University Press. http://books. google.it/books?id=YZRODCVYACsC

John C. McCarthy. 1986. Mental Situation Calculus. In Proceedings of the 1986 Conference on Theoretical Aspects of Reasoning About Knowledge (TARK '86). Morgan Kaufmann Publishers Inc., San Francisco, CA, USA, 307-307. http://dl.acm.org/ citation.cfm?id=1029786.1029815

G.A. Miller. 1995. WordNet: a lexical database for English. Commun. ACM 38, 11 (1995), 39-41.

Milind Naphade, John R. Smith, Jelena Tesic, Shih-Fu Chang, Winston Hsu, Amd Lyndon Kennedy, Alexander Hauptmann, and Jon Curtis. 2006. Large-scale concept ontology for multimedia. IEEE Multimedia 13, 3 (2006), 86-91.

E. Norling and L. Sonenberg. 2004. Creating Interactive Characters with BDI Agents. In Proceedings of the Australian Workshop on Interactive Entertainment IE2004.

O' Neill, E. T. 2002. FRBR: Functional Requirements for Bibliographic Records; Application of the Entity-Relationship Model to Humphry Clinker. Library Resourches and Technical Services 46 (2002), 150-158.

A. Pease, I. Niles, and J. Li. 2002. The suggested upper merged ontology: A large ontology for the semantic web and its applications. In Working Notes of the AAAI-2002 Workshop on Ontologies and the Semantic Web, Vol. 28. Edmonton, Canada.

F. Peinado, M. Cavazza, and D. Pizzi. 2008. Revisiting Character-based Affective Storytelling under a Narrative BDI Framework. In Proc. of ICIDIS08. Erfurt, Germany.

John Pemble. 2005. Shakespeare Goes to Paris: How the Bard Conquered France. Bloomsbury Academic. https://books.google. it/books?id=DH1IRnaUOokC

Manfred Pfister. 1991. The Theory and Analysis of Drama. Cambridge University Press. http://books.google.it/books?id= 2BxOGkheiHMC

Emanuele Pianta, Luisa Bentivogli, and Christian Girardi. 2002. MultiWordNet: developing an aligned multilingual database. In Proceedings of the First International Conference on Global WordNet. http://multiwordnet.fbk.eu/paper/ MWN-India-published.pdf

Georges Polti. 1895. Les trente-six situations dramatiques. Mercure de France, Paris.

Allen H Renear and David Dubin. 2007. Three of the four FRBR Group 1 entity types are roles, not types. Proceedings of the American Society for Information Science and Technology 44, 1 (2007), 1-19.

Jenn Riley. 2008. Application of the Functional Requirements for Bibliographic Records (FRBR) to Music. ISMIR (2008). http://books.google.com/books?hl=en $\backslash \& l \mathrm{r}=\backslash \& \mathrm{id}=\mathrm{OHp3sRnZD-oC} \backslash \&$ oi =fnd $\backslash \& p g=P A 439 \backslash \& \mathrm{dq}=\mathrm{APPLICATION}+\mathrm{OF}+\mathrm{THE}+$ FUNCTIONAL+REQUIREMENTS+FOR+BIBLIOGRAPHIC+RECORDS+(+FRBR+)+TO+MUSIC $\backslash \&$ ots=oELMnIgye8 \&sig=8S2UtDXOI18yKcUDpFh6baLpPfs

Marie Laure Ryan. 2006. Avatars of Story. University of Minnesota Press.

Jean-Pierre Ryngaert. 2008. Introduction à l'analyse du théâtre. Armand Colin. http://books.google.it/books?id=WldsIAAACAAJ

ACM Journal on Computing and Cultural Heritage, Vol. 2, No. 3, Article 1, Publication date: May 2010. 
Simone Sacchi, Karen Wickett, Allen Renear, and David Dubin. 2011. A framework for applying the concept of significant properties to datasets. Proceedings of the American Society for Information Science and Technology 48, 1 (2011), 1-10.

Sebastian Sardina, Lavindra de Silva, and Lin Padgham. 2006. Hierarchical planning in BDI agent programming languages: A formal approach. In Proceedings of the fifth international joint conference on Autonomous agents and multiagent systems. ACM, 1001-1008.

Ranisha Singh. 2013. Existential Shakespeare in Postmodern Love: An Analysis of Hamlet and Romeo and Juliet Through Film and Text. PhD. Dissertation. Iona College, New Rochelle, NY. https://books.google.it/books?id=vsx0ngEACAAJ

Rieks Smeets. 2004. Intangible Cultural Heritage and Its Link to Tangible Cultural and Natural Heritage. In Okinawa International Forum 2004 UTAKI in Okinawa and Sacred Spaces in Asia: Community Development and Cultural Heritage, Masako Yamamoto and Mari Fujimoto (Eds.). The Japan Foundation, 137-150.

L. Smith and N. Akagawa. 2008. Intangible Heritage. Taylor \& Francis. http://books.google.it/books?id=voan0ESUzgAC

Stuart Spencer. 2002. The Playwright's Guidebook: An Insightful Primer on the Art of Dramatic Writing. Faber \& Faber. http://books.google.it/books?id=nDrHmckSqi4C

F.M. Suchanek, G. Kasneci, and G. Weikum. 2007. Yago: a core of semantic knowledge. In Proceedings of the 16th international conference on World Wide Web. ACM, 697-706.

Peter Szondi. 1983. Theory of the Moderna Drama Parts I-II. boundary 2 11, 3 (Spring 1983), 191-230.

Y. Tanahashi and Kwan-Liu Ma. 2012. Design Considerations for Optimizing Storyline Visualizations. IEEE Transactions on Visualization and Computer Graphics 18, 12 (2012), 2679-2688. DOI :http://dx.doi.org/10.1109/TVCG.2012.212

Nicolas Yann. 2005. Folklore Requirements for Bibliographic Records: Oral Traditions and FRBR. In Functional requirements for bibliographic records (FRBR) : hype or cure-all?, Patrick. Le Boeuf (Ed.). Vol. 39. Haworth Information Press, Binghamton, NY, 179-195. https://www.researchgate.net/publication/228728033_Folklore_Requirements_for_Bibliographic_ RecordsOral_Traditions_and_FRBR

Received February 2015; revised July 2015; accepted August 2015 